Pacific Journal of Mathematics

REFLECTION LAWS OF SYSTEMS OF SECOND ORDER ELLIPTIC DIFFERENTIAL EQUATIONS IN TWO

CO Es 


\title{
REFLECTION LAWS OF SYSTEMS OF SECOND ORDER ELLIPTIC DIFFERENTIAL EQUATIONS IN TWO INDEPENDENT VARIABLES WITH CONSTANT COEFFICIENTS
}

\author{
JAMES M. SLOSS
}

In this paper we shall consider the reflection of solutions of systems of equations

$$
u_{x x}+u_{y y}+A u_{x}+B u_{y}+C u=0,
$$

where $u=\left(u_{1}, u_{2}, \cdots, u_{n}\right)^{T}, A, B, C$ are constant, pairwise commutative $n \times n$ matrices, across an analytic arc $\kappa$ on which the solutions satisfy $n$ analytic linear differential boundary conditions. If the boundary conditions have coefficients which are analyiic in a specific preassigned geometrical region cantaining $\kappa$, then we shall show that the solution of (1.1) satisfying such boundary conditions can be extended across $\kappa$, provided certain inequalities are satisfied. Moreover, the region into which $u$ can be extended will depend only on the analytic arc $\kappa$, the original region, and the coefficients of the boundary conditions; i.e., we shall have reflection "in the large" and the region will not be restricted by the equation.

There are two basically different situations considered, the results of which are stated in Theorem 1, Theorem 2, and Theorem 3.

Theorem 1 treats the reflection of solutions of a system (1.1) initially given on an open set $\Omega$ for which the boundary conditions on an arc $\kappa$ adjacent to $\Omega$ are

$$
\sum_{\beta=1}^{n} p_{\alpha \beta}(D) u_{\beta}=f_{\alpha}(z), \quad \alpha=1,2, \cdots, n \text { or } \kappa
$$

where

$$
p_{\alpha \beta}(D)=\sum_{r+s \leqq k<2 n}<p_{\alpha \beta}^{r s}(z) D_{x}^{r} D_{y}^{r}
$$

with $p_{\alpha \beta}^{r s}(z)$ and $f_{\alpha}(z)$ analytic in $\Omega \cup \kappa \cup \hat{\Omega}$, where $\hat{\Omega}$ is an open set determined by $\kappa$ adjacent to $\kappa$ and disjoint from $\Omega$. In the event that two inequalities involving the $p_{\alpha \beta}^{r s}(z)(r+s=k)$ are satisfied, then we can reflect the solution of the system across $\kappa$ into $\kappa \cup \hat{\Omega}$, so that the original solution is extended into all of $\Omega \cup \kappa \cup \hat{\Omega}$.

In Theorems 2 and 3 the reflection of solutions given in $\Omega$, of the special system (1.1)

$$
\Delta u+E u=0, \quad E=n \times n \text { constant matrix , }
$$


is treated. In these cases boundary conditions of the form

$$
\sum_{r+s \leqq k<2 n} p_{r 1}^{r s}(z) D_{x}^{r} D_{y}^{s} u_{1}=f_{\nu}(z), \quad \nu=1,2, \cdots, n
$$

are assumed prescribed on $k$, in which $p_{\nu 1}^{r s}(z)$ and $f_{\nu}(z)$ are analytic in $\Omega \cup \kappa \cup \hat{\Omega}$. For Theorem $2, k \geqq n$, and for Theorem $3, k=n-1$. There are five conditions which must be satisfied in Theorem 2 to insure reflection. Aside from two inequalities involving the $p_{v 1}^{r s}(z)$ that must be satisfied as in Theorem 1 , there is an additional determinental inequality on the arc $(z=\overline{G(z))}$

$$
\left|D^{\nu}[G(c)-G(z)]^{j}\right| \neq 0, \quad 1 \leqq \nu \leqq n-1, \quad 1 \leqq j \leqq n-1,
$$

which must be satisfied as well as two additional inequalities which depend on the constants of the differential equations.*

In Theorem 3 it is unnecessary to assume (1) one of the differential equation conditions, and (2) condition (1.2). Moreover, in Theorem 3 the reflection is reduced in quadratures whereas in Theorem 2 , for the general case, we have only an existential proof.

Finally, we shall give equations and boundary conditions to which the theorems apply. Theorem 1 is applicable when the boundary conditions are $u=\left(\varphi_{1}(z), \varphi_{2}(z), \cdots, \varphi_{n}(z)\right)$.

Theorem 2 and Theorem 3 are suitable for systems of differential equations of the form

$$
\sum_{j=1}^{m} P_{i j}(\Delta) u_{j}=0, \quad i=1,2, \cdots, m
$$

where the $P_{i j}$ are polynomials with constant coefficients and $\Delta$ is the Laplacian. Two inequalities involving the coefficients of the $P_{i j}$ must be satisfied. A special example is the metaharmonic equation

$$
\Delta^{n} u+a_{1} \Delta^{n-1} u+\cdots+a_{n} u=0, \quad a_{j}=\text { constant . }
$$

In this case it is only necessary to check one inequality for the differential equation in the case of Theorem 2. A special example of the metaharmonic equation is the polyharmonic equation

$$
\Delta^{n} u=0 \text {. }
$$

In this case there are no inequalities for the differential equation that must be checked for Theorem 2 or 3 . Also in this case there is a particularly simple representation of the solution in terms of analytic functions in $\Omega$ and analytic functions in $\hat{\Omega}$ which is a generalization of the representation in [8].

In the special case of equations

* J. Leray kindly pointed out to me that (62) and one of the d.e. inequalities are always satisfied and that the 3 holds for $k<2 n$. 


$$
\Delta u_{j}=a_{j 2} u_{1}+a_{j 2} u_{2} \quad j=1,2, \quad a_{j i}=\text { constants },
$$

a special case of which is the metaharmonic equation

$$
\Delta^{2} u+a \Delta u+b u=0, \quad a, b \text { constant , }
$$

the condition on the arc $\kappa$ is automatically satisfied. Moreover, the conditions of Theorem 2, for the biharmonic equation, reduce to the conditions given in [8] with the exception that Theorem 2 requires $u \in C^{2+k}(\Omega \cup \kappa) \cap C^{4}(\Omega)$ whereas [8] requires only that

$$
u \in C^{k}(\Omega \cup \kappa) \cap C^{4}(\Omega) \cap C^{2}(\Omega \cup \kappa) .
$$

Finally, it is noted that in the case when the analytic arc is a portion of the $x$ axis then the condition (1.2) is automatically satisfied.

Restricting ourselves to equations of the type (1.1) we get explicit representations for the solutions in terms of the zero order matrix Bessel function. For purposes of brevity we shall consider homogeneous equations (1.1) since the treatment of nonhomogeneous equations involves only obvious changes.

In his beautiful paper [6], Lewy thoroughly considered the question of a single elliptic equation in two independent variables for which the coefficients are analytic functions in a neighborhood of $\kappa$. Brown [1] considered the reflection laws for a general fourth order elliptic equation, with constant coefficients, in two independent variables across a straight line segment on which he assumes the solution satisfies two boundary conditions of the form

$$
\sum_{r+s \leqq 3} p_{\nu}^{r s} D_{x}^{r} D_{y}^{s} u(x, 0)=f_{\nu}(x), \quad \nu=1,2,
$$

where the line of reflection is $y=0$ and $p_{\nu}^{r s}$ are constants. Assuming the original domain is convex then he achieves reflection in the large, i.e., the domain of reflection is determined initially by the differential equation. Filipenko [2] investigated reflection for the harmonic equation in more than two independent variables across the plane $x_{1}=0$ and has shown that reflection in the large for certain initial domains is possible provided boundary conditions of the form

$$
\frac{\partial u}{\partial x_{1}}+P\left(x_{2}, x_{3}, \cdots, x_{n}\right) u=0
$$

are prescribed on the plane, where $P$ is a polynomial. Lewy [7] has given an example to show that the modification of $P$ from a polynomial to an analytic function is not possible. Garabedian [3], [4] has also investigated certain reflection laws in the small for a nonlinear elliptic equation and for quasilinear equations with special boundary conditions. J. Leray [5] has, in a very interesting paper, used reflection for the 
explicit determination of the Greens function for an $M$-harmonic equation in a band, when differential boundary conditions are given on the boundary of the band.

2. Geometric reflection across an analytic arc. Let $\kappa$ be an open analytic arc defined by the real analytic function $F(x, y)=0$ with $F_{x}^{2}+F_{y}^{2} \neq 0$. As shown in [8], this defines a function $\zeta=G(z)$, of the complex variable $z=x+i y$ which is analytic in a neighborhood of $\kappa$ and for which $\kappa$ is described by $z=\overline{G(z)} . \quad \hat{z}=\overline{G(z)}$ is called the reflection of $z$ across $\kappa$. $\hat{z}=z$ on $\kappa$. Let $\Omega$ be a semi-neighborhood of $\kappa$, with $G(z)$ analytic and univalent on $\Omega$ and thus $G^{\prime}(z) \neq 0$ on $\Omega$. Let $\hat{\Omega}=\overline{G(\Omega)}$ and assume $\hat{\Omega} \cap \Omega=\varnothing$. Then it can be shown that for $z$ in $\Omega \cup \kappa \cup \hat{\Omega}, G(z)$ is univalent, $\widehat{\hat{z}}=z$ and $G^{\prime}(z) \neq 0$. Moreover

$$
\overline{G^{\prime}(z)}=\left[G^{\prime}(\hat{z})\right]^{-1}
$$

and

$$
\overline{G^{\prime \prime}(z)}=-G^{\prime \prime}(\widehat{z}) G^{\prime}(\widehat{z})^{-3}
$$

3. Representation of the solutions. In this section we shall derive a representation for the solutions of (1.1) which are in $C^{\prime}(\Omega \cup \kappa)$. This will be done by a slight variant of the very elegant method developed by Lewy [6]. The solutions are expressed by means of a complex Riemann function, which can be found explicitly in our case.

First we consider the transformation

$$
u=e^{-(1 / 2)(A x+B y)} w(x, y)
$$

where the exponential matrix is defined as usual by its McLaurin expansion. Due to the pairwise commutativity of $A, B$ and $C$ we get (1.1) becomes

$$
e^{-(1 / 2)(A x+B y)}\left\{w_{x x}+w_{y y}+\frac{1}{4}\left[4 C-A^{2}-B^{2}\right] w\right\}=0,
$$

which is equivalent to:

$$
w_{x x}+w_{y y}+D w=0
$$

where

$$
D=\frac{1}{4}\left[4 C-A^{2}-B^{2}\right] .
$$

Note that (3.3) can be written for $z$ in $\Omega$ as

$$
4 \overline{G^{\prime}(z)} w_{z \hat{z}}+D w=0
$$

where 


$$
\frac{\partial}{\partial z}=\frac{1}{2}\left[\frac{\partial}{\partial x}-i \frac{\partial}{\partial y}\right], \frac{\partial}{\partial \widehat{z}}=\frac{1}{2}\left[\overline{G^{\prime}(z)}\right]^{-1}\left[\frac{\partial}{\partial x}+i \frac{\partial}{\partial y}\right]
$$

Let

$$
w(x, y)=w\left[\frac{z+\bar{z}}{2}, \frac{z-\bar{z}}{2 i}\right]=w\left[\frac{z+G(\hat{z})}{2}, \frac{z-G(\widehat{z})}{2 i}\right]=W(z, \widehat{z})
$$

for $z=x+i y \in \Omega \cup \kappa$ and $\widehat{z}=\overline{G(z)} \in \hat{\Omega} \cup \kappa$.

With the idea of finding a representation of the solution of (3.3), we seek the complex Riemann function; viz. the solution of

$$
L[v]=v_{z \zeta}+\frac{1}{4} D G^{\prime}(\zeta) v=0
$$

which is a function $R\left[z^{0}, \zeta^{0}, z, \zeta\right]$ of four complex arguments each ranging independently over $\Omega \cup \kappa \cup \hat{\Omega}$ with

$$
L_{z, \zeta}[R]=0
$$

and

$$
\begin{aligned}
& R\left[z^{0}, \zeta^{0} ; z^{0}, \zeta^{0}\right]=I \\
& R_{z}\left[z^{0}, \zeta^{0} ; z, \zeta^{0}\right]=0 . \\
& R_{\zeta}\left[z^{0}, \zeta^{0} ; z^{0}, \zeta\right]=0
\end{aligned}
$$

We claim that such a matrix function is given by

$$
R\left[z^{0}, \zeta^{0} ; z, \zeta\right]=J_{0}\left[\sqrt{\left.\overline{D\left(\left(z-z^{0}\right)\left(G(\zeta)-G\left(\zeta^{0}\right)\right)\right.}\right]}\right.
$$

where if $Q$ is an $n \times n$ matrix, we define

$$
J_{0}[\sqrt{Q}]=I-\frac{Q}{2^{2}}+\frac{Q^{2}}{2^{4}(2 !)^{2}}-\frac{Q^{3}}{2^{6}(3 !)^{2}}+\cdots .
$$

With any norm for $Q$ we get

$$
\left\|J_{0}[\sqrt{\bar{Q}}]\right\| \leqq J_{0}[i \sqrt{\|\bar{Q}\|}]
$$

where $J_{0}$, on the right, is the zero order Bessel function and thus the matrix series converges for all $Q$. Thus $R\left[z^{0}, \zeta^{0} ; z, \zeta\right]$, as defined, is analytic in $z^{0}, \zeta^{0}, z$ and $\zeta$ for $z^{0}, \zeta^{0}, z$ and $\zeta$ in $\Omega \cup \kappa \cup \hat{\Omega}$. Moreover it is easy to see that (3.9) are satisfied and by direct computation, we see that (3.8) is satisfied.

Our next aim is to find a representation of $W(z, \widehat{z})$ as defined by (3.6). This will be done by finding a function $W^{*}(z, \zeta)$ which is analytic for $z$ in $\Omega$ and analytic for $\zeta$ in $\hat{\Omega}$ and for which

$$
W^{*}(z, \hat{z})=W(z, \hat{z}) \text {. }
$$


We consider now the Cartesian product

$$
S \times \hat{S}=\{(z, \zeta): z \in \Omega \cup \kappa, \zeta \in \hat{\Omega} \cup \kappa\} .
$$

Let $z_{1}$ and $z_{2}$ be arbitrary points of $\Omega \cup \kappa$ and let $p$ be a path joining $z_{1}$ to $z_{2}$ which lies in $\Omega \cup \kappa$. Then let $\hat{p}$ be the reflection of $p$ joining $\widehat{z}_{1}$ to $\widehat{z}_{2}$ in $\hat{\Omega} \cup \kappa$. Let

$$
S^{2}\left(z_{1}, z_{2}, p\right)=\{(z, \zeta) \in S \times \hat{S}: z \in p \text { and } \zeta \in \hat{p}\} .
$$

Note that

$$
\begin{aligned}
R L\left[W^{*}\right]-L[R] W^{*} \\
\quad=R W_{z \zeta}^{*}+\frac{1}{4} G^{\prime}(\zeta) R D W^{*}-R_{z \zeta} W^{*}-\frac{1}{4} G^{\prime}(\zeta) D R W^{*} \\
\quad=\left(R W^{*}\right)_{z \zeta}-\left(R_{z} W^{*}\right)_{\zeta}-\left(R_{\zeta} W^{*}\right)_{z}
\end{aligned}
$$

since, as is clear from (3.10), $R D=D R$. We define

$$
\begin{aligned}
W^{*}(z, \hat{z})= & W(z, \hat{z})=w(x, y),\left.W_{z}^{*}(z, \zeta)\right|_{\zeta=\hat{z}}=W_{z}(z, \hat{z}), \\
& \left.W_{\zeta}^{*}(z, \zeta)\right|_{\zeta=\hat{z}}=W_{\hat{z}}(z, \hat{z}),
\end{aligned}
$$

i.e. the solution to equation (3.3), and let this be the "initial condition" for the extension of $W^{*}$ as an analytic function in $(z, \zeta)$. Let $W^{*}$ be assumed to be a solution of $L\left[W^{*}\right]=0$ for $(z, \zeta) \in S^{2}\left(z_{1}, z_{2}, p\right)$. We shall want to integrate (3.11), when $W^{*}$ is such a solution and $R$ is a Riemann function, over "triangles" $\Delta_{1}$ of $S^{2}\left(z_{1}, z_{2}, p\right)$ with vertices $\left(\hat{\zeta}^{0}, \zeta^{0}\right),\left(z^{0}, \zeta^{0}\right)$ and $\left(z^{0}, \hat{z}^{0}\right)$, over "triangles" $\Delta_{2}$ with vertices $\left(\hat{\zeta}^{0}, \zeta^{0}\right),\left(\hat{\zeta}^{0}, \hat{z}^{0}\right)$ and $\left(z^{0}, \hat{z}^{0}\right)$ and over "squares" with vertices $(c, \hat{c}),(z, \hat{c}),(z, \hat{z}),(c, \hat{z})$, $c$ being a point of $\kappa$. Over such "regions" as these, we have:

$$
0=-\oint\left(R W^{*}\right)_{t} d t+\oint R_{t} W^{*} d t-\oint R_{\sigma} W^{*} d \sigma .
$$

Consider $R\left[z^{0}, \zeta^{0}, t, \sigma\right]$ and $W^{*}(t, \sigma)$ in the above, where the region is the "triangle" $\subset S^{2}\left(z_{1}, z_{2}, p\right)$ with vertices $\left(\hat{\zeta}^{0}, \zeta^{0}\right),\left(z^{0}, \zeta^{0}\right),\left(z^{0}, \widehat{z}^{0}\right)$. We get, due to the nature of $R\left[z^{0}, \zeta^{0} ; t, \sigma\right]$,

$$
\begin{aligned}
W^{*}\left(z^{0}, \zeta^{0}\right)= & W^{*}\left(\hat{\zeta}^{0}, \zeta^{0}\right) \\
& +\int_{\left(\hat{\zeta}^{0}, \zeta^{0}\right)}^{\left(z^{0}, \hat{z}^{0}\right)}\left\{\left(R\left[z^{0}, \zeta^{0} ; t, \hat{t}\right] W^{*}[t, \hat{t}]\right)_{t}-R_{t} W^{*}\right\} d t \\
& +\int_{\left(\hat{\zeta}^{0}, \zeta^{0}\right)}^{\left(z^{0}, \hat{z}^{0}\right)} R_{\sigma}\left[z^{0}, \zeta^{0} ; \hat{\sigma}, \sigma\right] W^{*}(\hat{\sigma}, \sigma) d \sigma .
\end{aligned}
$$

Next we consider $R\left[\hat{\zeta}^{0}, \hat{z}^{0} ; t, \sigma\right]$ and $W^{*}(t, \sigma)$ in (3.12) and integrate over the triangle $\subset S^{2}\left(z_{1}, z_{2}, p\right)$ with vertices $\left(\hat{\zeta}^{0}, \zeta^{0}\right),\left(z^{0}, \hat{z}^{0}\right),\left(\hat{\zeta}^{0}, \hat{z}^{0}\right)$ and get, making use of the special character of the Riemann function 


$$
\begin{aligned}
W^{*}\left(\hat{\zeta}^{0}, \hat{z}^{0}\right)= & W^{*}\left(z^{0}, \hat{z}^{0}\right) \\
& -\int_{\left(\hat{\zeta}^{0} \zeta^{0}\right)}^{\left(z^{0}, \hat{z}^{0}\right)}\left\{\left(R\left[\hat{\zeta}^{0}, \widehat{z}^{0} ; t, \hat{t}\right] W^{*}(t, \hat{t})\right)_{t}-R_{t} W^{*}\right\} d t \\
& -\int_{\left(\hat{\zeta}^{0} \zeta^{0}\right)}^{\left(z^{0} \hat{z}^{0}\right)} R_{\sigma}\left[\hat{\zeta}^{0}, \widehat{z}^{0} ; \hat{\sigma}, \sigma\right] W^{*}(\hat{\sigma}, \sigma) d \sigma .
\end{aligned}
$$

Finally we shall integrate (3.12) with $R[z, \widehat{z} ; t, \sigma]$ over the rectangle $\subset S^{2}\left(z_{1}, z_{2}, p\right)$ with vertices $(c, c),(z, c),(z, \widehat{z}),(c, \widehat{z})$ where $c$ is assumed to be a point of $\kappa$, and thus, $(c=\hat{c})$ :

$$
\begin{aligned}
W^{*}(z, \widehat{z})= & W^{*}(c, \hat{z})+W^{*}(z, c)-R[z, \hat{z} ; c, c] W^{*}(c, c) \\
& -\int_{(c, c)}^{(z, c)} R_{t}[z, \hat{z} ; t, c] W^{*}(t, c) d t \\
& -\int_{(c, c)}^{(c, \hat{z})} R_{\sigma}[z, \hat{z} ; c, \sigma] W^{*}(c, \sigma) d \sigma .
\end{aligned}
$$

This gives the representation of the solution of (3.3) for which we were looking. The integrals entering (3.13), (3.14) and (3.15) are independent of the path $p$ since in (3.13) and (3.14)

$$
\begin{aligned}
{\left[\left(R W^{*}\right)_{t}-\left(R_{t} W^{*}\right)\right]_{\sigma}-\left[R_{\sigma} W^{*}\right]_{t} } & =R_{\sigma} W_{t}^{*}+R W_{t \sigma}^{*}-R_{\sigma t} W^{*}-R_{\sigma} W_{t}^{*} \\
& =R L_{t a}\left[W^{*}\right]-L_{\sigma t}[R] W^{*}=0
\end{aligned}
$$

by (3.11).

Next we show that $W^{*}(z, \zeta)$ as defined by (3.13) is an analytic function of $z$ and $\zeta$ for $z$ in $\Omega$ and $\zeta \in \hat{\Omega}$. This is done by showing $\partial W^{*}(z, \zeta) / \partial \bar{z}=0$ and $\partial W^{*}(z, \zeta) / \partial \bar{\zeta}=0$; i.e. the Cauchy Riemann equations are satisfied. Since $R$ is an analytic function of its arguments, $\widehat{\hat{z}}=z, \widehat{z}=\overline{G(z)}, d \hat{z} / d \bar{z}=\overline{G^{\prime}(z)}$,

$$
\left.\overline{G^{\prime}(z)} W^{*}(z, \widehat{z}) R_{\sigma}(z, \zeta ; \hat{\sigma}, \sigma)\right|_{\hat{\sigma}=z, \sigma=\hat{z}}=0
$$

by the nature of $R$. Next we check analyticity in $\zeta$.

$$
\begin{gathered}
\overline{G^{\prime}(\zeta)} W_{\hat{\zeta}} *(\hat{\zeta}, \zeta)-\overline{G^{\prime}(\zeta)}\left[\left(R(z, \zeta ; t, \hat{t}) W^{*}(t, \hat{t})\right)_{t}-R_{t} W^{*}(t, \hat{t})\right]_{t=\hat{\zeta} \hat{t}=\zeta} \\
=\overline{G^{\prime}(\zeta)}\left[W_{\hat{\zeta}}^{*}(\hat{\zeta}, \zeta)-\left.R(z, \zeta ; t, \hat{t}) W_{t}^{*}(t, \hat{t})\right|_{t=\hat{\zeta} \hat{t}=\zeta}\right]=0
\end{gathered}
$$

by the nature of $R=J_{0}$. Note that (3.14) can be got from (3.13) simply by substitution. The representation of the solutions of (1.1), which we shall use, is given with the aid of (3.1) and (3.15) by:

$$
\begin{aligned}
U^{*}(z, \widehat{z})= & \exp \left\{A^{*} z+B^{*} G(\widehat{z})\right\}\left\{W^{*}(z, c)+W^{*}(c, \widehat{z})\right. \\
& -R[z, \widehat{z} ; c, c] W^{*}(c, c) \\
& -\int_{c}^{z} R_{t}[z, \hat{z} ; t, c] W^{*}(t, c) d t \\
& \left.-\int_{c}^{\hat{z}} R_{o}[z, \hat{z} ; c, \sigma] W^{*}(c, \sigma) d \sigma\right\}
\end{aligned}
$$


where

$$
A^{*}=-\frac{1}{4}(A-i B), \quad B^{*}=-\frac{1}{4}(A+i B) .
$$

4. Reflection of solutions of (1.1) across analytic boundary conditions. Before proving the reflection theorems we shall need to prove two lemmas.

LEMma 1. Let $\mu=\mu_{1}+\mu_{2}, \mu_{1}$ and $\mu_{2}$ nonnegative integers,

$$
D_{z}=\frac{1}{2}\left(D_{x}-i D_{y}\right), \quad D_{\hat{z}}=\frac{1}{2} G^{\prime}(\widehat{z})\left(D_{x}+i D_{y}\right)
$$

then for functions

$$
M(x, y)=M\left[\frac{z+G(\widehat{z})}{2}, \frac{z-G(\hat{z})}{2 i}\right]=M^{*}(z, \hat{z})
$$

that are analytic in $x$ and $y$, the following operator relation holds:

$$
\begin{aligned}
D_{x}^{\mu_{1}} D_{y}^{\mu_{2}}= & (i)^{\mu_{2}}\left\{\sum_{j=0}^{\mu} \alpha_{j^{1}}^{\mu_{2}}\left(G^{\prime}(\widehat{z})\right)^{-j} D_{z}^{\mu-j} D_{\hat{z}}^{j}-\delta(2, \mu) \alpha_{\mu}^{\mu_{1} \mu_{2}}(\mu-2)\right. \\
& \cdot\left(G^{\prime}(\widehat{z})\right)^{-(\mu+1)}\left(G^{\prime \prime}(\hat{z})\right) D_{\hat{z}}^{\mu-1} \\
& + \text { terms of order } \leqq \mu-1 \text { in } D_{z} \text { and } D_{\hat{z}} \text { all } \\
& \text { of which contain terms of order at least } \\
& \text { one and not greater than } \mu-2 \text { in } D_{\hat{z}} ; \text { i.e. } \\
& \text { all of order } \left.\leqq \mu-2 \text { in } D_{z}\right\}
\end{aligned}
$$

where $\alpha_{0}^{\mu_{1} \mu_{2}}, \alpha_{1}^{\mu_{1} \mu_{2}}, \alpha_{2}^{\mu_{1} \mu_{2}}, \cdots, \alpha_{\mu}^{\mu_{1} \mu_{2}}$ are the coefficients of $a^{\mu}, a^{\mu-1} b, \cdots, b^{\mu}$ in $(a+b)^{\mu_{1}}(a-b)^{\mu_{2}}\left(\right.$ Note $\left.\alpha_{0}^{\mu_{1} \mu_{2}}=1, \alpha_{\mu}^{\mu_{1} \mu_{2}}=(-1)^{\mu_{2}}\right)$ and

$$
\begin{aligned}
\delta(\nu, \mu) & =0 \text { if } \nu>\mu, \\
& =1 \text { if } \nu \leqq \mu .
\end{aligned}
$$

Proof. By induction on $\mu$. For $\mu=0$ clearly true. For $\mu=1$ we have from (4.1) for $\mu_{1}=1, \mu_{2}=0$

$$
D_{x}=D_{z}+\left(G^{\prime}(\widehat{z})\right)^{-1} D_{\hat{z}} \quad \text { which is }(4.2) \text { for } \mu_{1}=1, \mu_{2}=0
$$

and for $\mu_{1}=0, \mu_{2}=1$

$$
D_{y}=i D_{z}-i\left(G^{\prime}(\hat{z})\right)^{-1} D_{\hat{z}} \quad \text { which is (4.2) for } \mu_{1}=0, \mu_{2}=1 .
$$

Assume (4.2) true for $\mu$, we must show it is ture for $\mu+1$. i.e. assume (4.2) and then consider 
(A)

$$
\begin{aligned}
D_{x}^{\mu_{1}+1} D_{y}^{\mu_{2}}= & (i)^{\mu_{2}}\left\{\sum_{j=0}^{\mu} \alpha_{j}^{\mu_{1} \mu_{2}}\left[\left(G^{\prime}(\hat{z})\right)^{-j} D_{z}^{\mu+1-j} D_{\hat{z}}^{j}+\left(G^{\prime}(\widehat{z})\right)^{-(j+1)} D_{z}^{\mu-j} D_{\hat{z}}^{j+1}\right]\right. \\
& -\alpha_{\mu}^{\mu_{1} \mu_{2}}\left(G^{\prime}(\hat{z})\right)^{-(\mu+2)} G^{\prime \prime}(\hat{z})[\mu+(\mu-2)] D_{\hat{z}}^{\mu}
\end{aligned}
$$

A) $\quad+$ terms of order $\leqq \mu$ in $D_{z}$ and $D_{\hat{z}}$ all containing terms of order at least one and not greater than $\mu-1$ in $\left.D_{\hat{z}}\right\}$. i.e.,

$$
\begin{aligned}
D_{x}^{\mu_{1}+1} D_{y}^{\mu_{2}}= & (i)^{\mu_{2}}\left\{D_{z}^{\mu+1}+\sum_{j=1}^{\mu}\left(G^{\prime}(\widehat{z})\right)^{-j} D_{z}^{\mu+1-j} D_{\hat{z}}^{j}\left[\alpha_{j}^{\mu_{1} \mu_{2}}+\alpha_{j-1}^{\mu_{1} \mu_{2}}\right]\right. \\
& +\alpha_{\mu}^{\mu_{1} \mu_{2}}\left(G^{\prime}(\widehat{z})\right)^{-(\mu+1)} D_{\widehat{z}}^{\mu+1} \\
& \left.-\alpha_{\mu}^{\mu_{1} \mu_{2}}\left(G^{\prime}(\widehat{z})\right)^{-(\mu+2)} G^{\prime \prime}(\widehat{z})\left(\begin{array}{c}
\mu+1 \\
\mu-1
\end{array}\right) D_{\hat{z}}^{\mu}+\cdots\right\} .
\end{aligned}
$$

But

$$
\begin{aligned}
(a+b) & (a+b)^{\mu_{1}}(a-b)^{\mu_{2}} \\
= & (a+b)\left\{a^{\mu}+\alpha_{1}^{\mu_{1} \mu_{2}} a^{\mu-1} b+\alpha_{2}^{\mu_{1} \mu_{2}} a^{\mu-2} b^{2}+\cdots+\alpha_{\mu}^{\mu_{1} \mu_{2}} b^{\mu}\right\} \\
= & a^{\mu+1}+\left(\alpha_{1}^{\mu_{1} \mu_{2}}+1\right) a^{\mu} b+\left(\alpha_{1}^{\mu_{1} \mu_{2}}+\alpha_{2}^{\mu_{1} \mu_{2}}\right) a^{\mu-1} b^{2} \\
& +\cdots+\left(\alpha_{\mu-1}^{\mu_{1} \mu_{2}}+\alpha_{\mu}^{\mu_{1} \mu_{2}}\right) a b^{\mu} \\
& +\alpha_{\mu}^{\mu_{1} \mu_{2}} b^{\mu+1}
\end{aligned}
$$

and thus we see

$$
\begin{aligned}
D_{x}^{\mu_{1}+1} D_{y}^{\mu_{2}}= & (i)^{\mu_{2}}\left\{\sum_{j=0}^{\mu+1} \alpha_{j}^{\mu_{1}+1 \mu_{2}}\left(G^{\prime}(\widehat{z})\right)^{-j} D_{z}^{\mu+1-j} D_{\hat{z}}^{j}\right. \\
& -\alpha_{\mu+1}^{\mu_{1}+1 \mu_{2}}\left(G^{\prime}((\widehat{z}))^{-(\mu+2)} G^{\prime \prime}(\hat{z})\left(\begin{array}{l}
\mu+1 \\
\mu-1
\end{array}\right) D_{\hat{z}}^{\mu}+\cdots\right\}
\end{aligned}
$$

where $\alpha_{j}^{\mu_{1}+1 \mu_{2}}$ are the coefficients of $a^{\mu+1}, a^{\mu} b, \cdots, b^{\mu+1}$ in $(a+b)^{\mu_{1}+1}(a-b)^{\mu_{2}}$. Now consider

$$
\begin{aligned}
D_{x}^{\mu_{1}} D_{y}^{\mu_{2}+1}= & (i)^{\mu_{2}+1}\left\{D_{z}^{\mu+1}+\sum_{j=1}^{\mu}\left(G^{\prime}(\widehat{z})\right)^{-j} D_{z}^{\mu+1-j} D_{\hat{z}}^{j}\left[\alpha_{j+1}^{\mu_{1} \mu_{2}}-\alpha_{j}^{\mu_{1} \mu_{2}}\right]\right. \\
& -\alpha_{\mu}^{\mu_{1} \mu_{2}}\left(G^{\prime}(\widehat{z})\right)^{-(\mu+1)} D_{\widehat{z}}^{\mu+1} \\
& \left.+\alpha_{\mu}^{\mu_{1} \mu_{2}}\left(G^{\prime}(\widehat{z})\right)^{-(\mu+2)} G^{\prime \prime}(\widehat{z})\left[\mu+\left(\begin{array}{c}
\mu \\
\mu
\end{array}\right)\right] D_{\hat{z}}^{\mu}+\cdots\right\} \\
= & (i)^{\mu_{2}+1}\left\{\sum_{j=0}^{\mu+1} \alpha_{j}^{\mu_{1} \mu_{2}+1}\left(G^{\prime}(\widehat{z})\right)^{-j} D_{z}^{\mu+1-j} D_{\hat{z}}^{j}\right. \\
& \left.-\alpha_{\mu+1}^{\mu_{1} \mu_{2}+1}\left(G^{\prime}(\widehat{z})\right)^{-(\mu+2)} G^{\prime \prime}(\widehat{z})\left(\begin{array}{l}
\mu+1 \\
\mu-1
\end{array}\right) D_{\hat{z}}^{\mu}+\cdots\right\}
\end{aligned}
$$

where since

$$
\begin{aligned}
(a-b)(a+b)^{\mu_{1}}(a-b)^{\mu_{2}}=a^{\mu+1} & +\left(\alpha_{1}^{\mu_{1} \mu_{2}}-1\right) a^{\mu} b+\left(\alpha_{2}^{\mu_{1} \mu_{2}}-\alpha_{1}^{\mu_{1} \mu_{2}}\right) a^{\mu-1} b^{2} \\
& +\cdots+\left(\alpha_{\mu}^{\mu_{1} \mu_{2}}-\alpha_{\mu-1}^{\mu_{1} \mu_{2}}\right) a b^{\mu}-\alpha_{\mu}^{\mu_{1} \mu_{2}} b^{\mu+1}
\end{aligned}
$$

the $\alpha_{j}^{\mu_{1} \mu_{2}}$ are the coefficients of $a^{\mu+1}, a^{\mu} b, \cdots b^{\mu+1}$ in $(a+b)^{\mu_{1}}(a-b)^{\mu_{2}+1}$. Thus the lemma is proved. 
LEMMA 2. Given the operators

$$
p_{i 1}(D)+p_{i 2}(D)+\cdots+p_{i n}(D) \quad i=1,2, \cdots, n
$$

where

$$
p_{i 1}(D)=\sum_{r+s \leqq k} p_{i 1}^{r s}(z) D_{x}^{r} D_{y}^{s}, p_{i 2}(D)=\sum_{r+s \leqq k} p_{i 2}^{r s}(z) D_{x}^{r} D_{y}^{s}, \cdots
$$

then for

$$
\begin{aligned}
D_{z}= & \frac{1}{2}\left(D_{x}-i D_{y}\right), D_{\hat{z}}=\frac{1}{2} G^{\prime}(\widehat{z})\left(D_{x}+i D_{y}\right) \\
\left(p_{i j}(D)\right)=M= & M_{k, 0}(z) D_{z}^{k}+M_{k-1,1}(z)\left(G^{\prime}(\widehat{z})\right)^{-1} D_{z}^{k-1} D_{\widehat{z}}+M_{k-1,0}(z) D_{z}^{k-1} \\
& +M_{0, k}(z)\left(G^{\prime}(\widehat{z})\right)^{-k} D_{\hat{z}}^{k}+M_{1, k-1}(z)\left(G^{\prime}(\widehat{z})\right)^{-(k-1)} D_{z} D_{\widehat{z}}^{k-1} \\
& +M_{0, k-1}(z) D_{\widehat{z}}^{k-1}+T_{k-2}(z)
\end{aligned}
$$

where $T_{k-2}(z)$ is a matrix of terms in $D_{z}$ and $D_{\hat{z}}$ and of order $\leqq k-2$ in $D_{z}$ and of order $\leqq k-2$ in $D_{\hat{z}}$.

$$
\begin{aligned}
& M_{k, 0}(z)=\sum_{r+s=k}(i)^{s}\left(\begin{array}{cc}
p_{11}^{r s} & p_{12}^{r s} \cdots p_{1 n}^{r s} \\
\vdots & \\
p_{n 1}^{r s} & \cdots p_{n n}^{r s}
\end{array}\right)=\sum_{r+s=k}(i)^{s}\left(p_{\alpha \beta}^{r s}\right) \\
& M_{k-1,1}(z)=\sum_{r+s=k}(i)^{s} \alpha_{1}^{r s}\left(p_{\alpha \beta}^{r s}\right), M_{k-1,0}(z)=\sum_{r+s=k-1}(i)^{s}\left(p_{\alpha \beta}^{r s}\right) \\
& M_{0, k}(z)=\sum_{r+s=k}(-i)^{s}\left(p_{\alpha \beta}^{r s}\right), M_{1, k-1}(z)=\sum_{r+s=k}(i)^{s} \alpha_{k-1}^{r s}\left(p_{\alpha \beta}^{r s}\right) \\
& M_{0, k-1}(z)=\sum_{r+s=k-1}(i)^{s} \alpha_{k-1}^{r s}\left(G^{\prime}(\widehat{z})\right)^{-(k-1)}\left(p_{\alpha \beta}^{r s}\right)-\delta(2, k) \\
& \times \sum_{r+s=k}(i)^{s} \alpha_{k}^{r s}\left(\begin{array}{c}
k \\
k-2
\end{array}\right)\left(G^{\prime}(\widehat{z})\right)^{-(k+1)}\left(G^{\prime \prime}(\widehat{z})\right)\left(p_{\alpha \beta}^{r s}\right)
\end{aligned}
$$

where $\alpha_{l}^{r s}$ are the same as in Lemma 1.

Proof. By Lemma 1

$$
\begin{aligned}
p_{\alpha 1}(D)= & \sum_{r+s=k} p_{\alpha 1}^{r s}\left\{( i ) ^ { s } \left[\sum_{j=0}^{k} \alpha_{j}^{r s}\left(G^{\prime}(\hat{z})\right)^{-j} D_{z}^{k-j} D_{\hat{z}}^{j}\right.\right. \\
& \left.\left.-\delta(2, k) \alpha_{k}^{r s}\left(\begin{array}{c}
k \\
k-2
\end{array}\right)\left(G^{\prime}(\widehat{z})\right)^{-(k+1)}\left(G^{\prime \prime}(\widehat{z})\right) D_{\widehat{z}}^{k-1}\right]\right\} \\
& +\sum_{r+s=k-1}(i)^{s} p_{\alpha 1}^{r s} D_{z}^{k-1}+\sum_{r+s=k-1}(i)^{s} p_{\alpha 1}^{r s} \alpha_{k-1}^{r s}\left(G^{\prime}(\widehat{z})\right)^{-(k-1)} D_{\hat{z}}^{k-1} \\
& +q_{i 1}\left(D_{z}, D_{\hat{z}}\right) \text { where } q_{i 1} \text { is a polynomial in } D_{z} \text { and } D_{\hat{z}}
\end{aligned}
$$

of degree $\leqq k-1$ with coefficients analytic in $\Omega \cup \kappa \cup \hat{\Omega}$ and which contains terms of order $\leqq k-2$ in $D_{z}$ and of order $\leqq k-2$ in $D_{\hat{z}}$. Similar results hold for $p_{\alpha \beta}(D), \beta=2, \cdots, n$. Combining these results with the fact that $\alpha_{k}^{r s}=(-1)^{s}$, we get the conclusion of the lemma. 
We are now in a position to prove

THEOREM 1. Let $u=\left(u_{1}, u_{2}, \cdots, u_{n}\right) \in C^{2}(\Omega)$ and satisfy in $\Omega$

$$
u_{x x}+u_{y y}+A u_{x}+B u_{y}+C u=0
$$

where $A, B, C$ are pairwise commutative constant $n \times n$ matrices. Moreover let $u \in C^{k}(\Omega \cup \kappa) \cap C^{\prime}(\Omega \cup \kappa)$ and satisfy on $\kappa$ the boundary conditions:

$$
\sum_{\beta=1}^{n} p_{\alpha \beta}\left(z, D_{x}, D_{y}\right) u_{\beta}=f_{\alpha}(z), \quad \alpha=1,2, \cdots, n,
$$

where

$$
p_{\alpha \beta}(z, \xi, \eta)=\sum_{r+s \leqq k<2 n} p_{\alpha \beta}^{r s}(z) \xi^{r} \eta^{s},
$$

with $p_{\alpha \beta}^{r s}(z)$ and $f_{\alpha}(z)$ analytic in $\Omega \cup \kappa \cup \hat{\Omega}$. Moreover, if $P_{\alpha \beta}(z, \xi, \eta)$ is the principal part of $p_{\alpha \beta}(z, \xi, \eta)$, we assume in $\Omega \cup \kappa \cup \hat{\Omega}$

$$
0 \neq\left|M_{k, 0}(z)\right|=\left|P_{\alpha \beta}(z, 1,-i)\right|^{1}
$$

and

$$
0 \neq\left|M_{0, k}(z)\right|=\left|P_{\alpha \beta}(z, 1,-i)\right|^{1} .
$$

Then we can reflect $u$ across $\kappa$ into $\hat{\Omega}$; i.e., there exists a unique function $u$ which is a solution of (1.1) in $\Omega \cup \kappa \cup \hat{\Omega}$ and which agrees with the given solutions $u$ of (1.1) in $\Omega \cup \kappa$.

Proof. We apply $M$ of Lemma 2 to the representation (3.16) and evaluate on $\kappa$. For simplicity let

$$
g(\widehat{z})=W^{*}(c, \hat{z}), \quad h(z)=W^{*}(z, c) .
$$

Then we get on $\kappa$, remembering that $z=\hat{z}$ there:

$$
e^{A^{*} z+B^{*} G(z)}\left\{M_{k, 0}(z) h^{(k)}(z)+M_{0, k}(z)\left(G^{\prime}(z)\right)^{-k} g^{(k)}(z)+T_{k-1}[g, h, z]\right\}=f(z),
$$
where $f(z)=\left(f_{1}(z), \cdots, f_{n}(z)\right)^{T}$ and $T_{k-1}[g, h, z]$ is an expression of the form:

$$
\begin{aligned}
T_{k-1}[g, h, z]= & a_{k-1}(z) h^{(k-1)}(z)+a_{k-2}(z) h^{(k-2)}(z)+\cdots+a_{0}(z) h(z) \\
& +\int_{c}^{z} a_{-1}(z, t) h(t) d t+b_{k-1}(z) g^{(k-1)}(z)+\cdots+b_{0}(z) g(z) \\
& +\int_{c}^{z} b_{-1}(z, t) g(t) d t+E(z) W^{*}(c, c)
\end{aligned}
$$

1 As Professor Jean Leray has kindly pointed out to me, these statements concern the behavior of the boundary conditions in the characteristic directions. 
where $E$ and the $a^{\prime}$ s and $b^{\prime}$ s are matrices, analytic in $\Omega \cup \kappa \cup \hat{\Omega}$. Note that for $j \geqq 1$,

$$
h(z)=\frac{1}{(j-1) !} \int_{c}^{z}(z-t)^{j-1} h^{(j)}(t) d t+\sum_{\sigma=0}^{j-1} \frac{1}{\sigma !}(z-c)^{\sigma} h^{(\sigma)}(c)
$$

and similarly for $g(z)$ where $h^{(\sigma)}(c)$ and $g^{(\sigma)}(c)$ are known via (3.13) and (3.14). Moreover if $K(z, t)$ is a matrix function known and analytic in $\Omega \cup \kappa \cup \hat{\Omega}$ then for $k \geqq 1$

$$
\int_{c}^{z} K(z, t) h(t) d t=\frac{1}{(k-1) !} \int_{c}^{z} d t_{1} K\left(z, t_{1}\right) \int_{c}^{t_{1}}\left(t_{1}-t_{2}\right)^{k-1} h^{(k)}\left(t_{2}\right) d t_{2}+K_{1}(z)
$$

where $K_{1}(z)$ is a known matrix function analytic in $\Omega \cup \kappa \cup \hat{\Omega}$ and thus

$$
\int_{c}^{z} K(z, t) h(t) d t=\int_{c}^{z} K_{2}(z, t) h^{(k)}(t) d t+K_{1}(z)
$$

where

$$
K_{2}(z, t)=\frac{1}{(k-1) !} \int_{t_{1}=t}^{z} d t_{1} K\left(z, t_{1}\right)\left(t_{1}-t\right)^{k-1} .
$$

Thus (4.5) becomes on $\kappa$ with the aid of (4.7) and (4.8) and the significance of (4.6).

$$
\begin{aligned}
& e^{A^{*} z+B^{*} G(z)}\left\{M_{k, 0}(z) h^{(k)}(z)+M_{0, k}(z)\left(G^{\prime}(z)\right)^{-k} g^{(k)}(z)\right. \\
& \left.\quad+\int_{c}^{z} K^{*}(z, t) h^{(k)}(t) d t+\int_{c}^{z} K^{* *}(z, t) g^{(k)}(t) d t+H(z)\right\}=f(z)
\end{aligned}
$$

where $H(z)$ is a known vector function of $z$, analytic in $\Omega \cup \kappa \cup \hat{\Omega}$, and $K^{*}$ and $K^{* *}$ are known matrix functions of $z, t$ analytic in $\Omega \cup \kappa \cup \hat{\Omega}$. Thus, since $\left|M_{k, 0}(z)\right| \neq 0$ and $\left|M_{0, k}(z)\right| \neq 0$ and $G^{\prime}(z) \neq 0$ in $\Omega \cup \kappa \cup \hat{\Omega}$, we can solve for $h^{(k)}(z)$ and $g^{(k)}(z)$ and get:

$$
h^{(k)}(z)=\int_{c}^{z} \widetilde{K}(z, t) h^{(k)}(t) d t+\widetilde{H}(z) \text { on } \kappa
$$

and

$$
g^{(k)}(z)=\int_{c}^{z} \widetilde{\widetilde{K}}(z, t) g^{(k)}(t) d t+\widetilde{\widetilde{H}}(z) \text { on } \kappa
$$

where $\widetilde{K}(z, t)$ and $\widetilde{\widetilde{K}}(z, t)$ are known matrix functions analytic for $z$ and $t$ in $\Omega \cup \kappa \cup \hat{\Omega}$ and $\widetilde{H}(z)$ is analytic in $\hat{\Omega}$ and continuous in $\hat{\Omega} \cup \kappa$ $\widetilde{\widetilde{H}}(z)$ is analytic in $\Omega$ and continuous in $\Omega \cup \kappa$.

Treating (4.10) as a system of Volterra integral equations in $\hat{\Omega} \cup \kappa$ and treating (4.11) as a system of Volterra integral equations in $\Omega \cup \kappa$, we get the analytic extension of $h^{(k)}(z)$ into $\Omega \cup \kappa \cup \hat{\Omega}$ and 
$g^{(k)}(z)$ into $\Omega \cup \kappa \cup \hat{\Omega}$. By integration, since $h$ and $g$ and their derivatives of order $\leqq k$ are known and continuous on $\kappa$ and specifically at $c$ at the outset, we get the unique extension of $h(z)$ and $g(z)$. By means of (3.15) we get the unique analytic extension of $W^{*}(z, \widehat{z})$ into $\Omega \cup \kappa \cup \hat{\Omega}$ and thus the extension of $u(x, y)$ into $\Omega \cup \kappa \cup \hat{\Omega}$.

We shall next concern ourselves with a system which is particularly useful when certain higher order equations are reduced to a system of equations. With this in mind, we shall consider a more restricted class of equations, since the inequality becomes very unwieldy.

Notation. Let

$$
E=\left(\begin{array}{c}
e_{1}^{1} \\
e_{2}^{1} \\
\vdots \\
e_{n}^{1}
\end{array}\right), E^{2}=\left(\begin{array}{c}
e_{1}^{2} \\
e_{2}^{2} \\
\vdots \\
e_{n}^{2}
\end{array}\right), \cdots, E^{k}=\left(\begin{array}{c}
e_{1}^{k} \\
e_{2}^{k} \\
\vdots \\
e_{n}^{k}
\end{array}\right)
$$

where $e_{m}^{j}=\left(e_{m 1}^{j}, e_{m 2}^{j}, \cdots, e_{m n}^{j}\right)$ is the $m^{\text {th }}$ row of $E^{j}$.

Before stating the theorem, we shall prove

LEMMA 3.

$$
\begin{aligned}
R[z, \zeta ; t, \sigma] & =p_{n-1}\{E,(t-z)[G(\sigma)-G(\zeta)]\} \\
& =\sum_{j=0}^{n-1} a_{j}\{(t-z)[G(\sigma)-G(\zeta)]\} E^{j}
\end{aligned}
$$

where $p_{n-1}(x, s)$ is the polynomial of degree $\leqq n-1$ in $x$ that interpolates $J_{0}[\sqrt{x s}]$ at the eigenvalues of $E$ (s held fixed); $a_{j}(s)$ are entire functions of $s$ and $E^{0}=I$. In the event that some or all of the eigenvalues $\lambda_{1}, \lambda_{2}, \cdots, \lambda_{j}$ of $E$ are multiple, i.e.,

$$
|E-\lambda I|=\left(\lambda-\lambda_{1}\right)^{n_{1}}\left(\lambda-\lambda_{2}\right)^{n_{2}} \cdots\left(\lambda-\lambda_{j}\right)^{n_{j}}(-1)^{n},
$$

$\lambda_{i} \neq \lambda_{k}$ if $i \neq k, n_{1}+n_{2}+\cdots+n_{j}=n$, then we use Hermite interpolation to determine $p_{n-1}(x)$ such that if $J(\lambda s)=J_{0}[\sqrt{\lambda s}]$

$$
\begin{array}{r}
p_{n-1}\left(\lambda_{1}, s\right)=J\left(\lambda_{1} s\right), \frac{\partial}{\partial \lambda} p_{n-1}\left(\lambda_{1}, s\right)=s J^{(1)}\left(\lambda_{1} s\right), \cdots, \frac{\partial^{n_{1}-1}}{\partial \lambda^{n_{1}-1}}, p_{n-1}\left(\lambda_{1}, s\right) \\
= \\
\begin{array}{r}
p_{n-1}\left(\lambda_{j}, s\right)=J\left(\lambda_{j} s\right), \frac{\partial}{\partial \lambda} p_{n-1}\left(\lambda_{j}, s\right)=s J^{(1)}\left(\lambda_{j} s\right), \cdots, \frac{\partial^{n_{j}-1}}{\partial \lambda^{n_{j}-1}}, p_{n-1}\left(\lambda_{j}, s\right) \\
=
\end{array} s^{n_{j}-1} J^{\left(n_{j}-1\right)}\left(\lambda_{j} s\right) .
\end{array}
$$

Proof. The unique Hermite interpolation polynomial $p_{n-1}(\lambda, s)$ is of the form: 


$$
\begin{aligned}
p_{n-1}(\lambda, s)=\sum_{i=1}^{j} J\left(\lambda_{i} s\right) l_{i 0}(\lambda) & +\sum_{i=1}^{j} s J^{(1)}\left(\lambda_{i} s\right) l_{i 1}(\lambda)+\cdots \\
& +\sum_{i=1}^{j} s^{n_{i-1}} J^{\left(n_{i}-1\right)}\left(\lambda_{i} s\right) l_{i\left(n_{i}-1\right)}(\lambda)
\end{aligned}
$$

where the $l_{i k}(\lambda)$ are polynomials in $\lambda$ of degree $\leqq n-1$.

Consider for $Q(\lambda)$ the characteristic polynomial of $E$ :

$$
f(\lambda, s)=\frac{J(\lambda s)-p_{n-1}(\lambda, s)}{Q(\lambda)} .
$$

$J(\lambda s)$ and $p_{n-1}(\lambda, s)$ are entire functions of $\lambda$ and $s$, moreover the polynomial $Q(\lambda)$ has the same zeros in $\lambda$ (multiplicity included) as $J(\lambda s)-p_{n-1}(\lambda, s)$. Thus $f(\lambda, s)$ is an entire function of $\lambda$ and $s$. Rearranging, we get

$$
J(\lambda s)=Q(\lambda) f(\lambda, s)+p_{n-1}(\lambda, s) .
$$

But $Q(\lambda)$ is the characteristic polynomial of $E$. Thus by the CayleyHamilton theorem $Q(E)=0$ and

$$
J(E s)=p_{n-1}(E, s)
$$

which gives the result since

$$
J\{E(t-z)[G(\sigma)-G(\zeta)]\}=J_{0}\{\sqrt{E(t-z)[G(\sigma)-G(\zeta)]}\}=R\{z, \zeta ; t, \sigma\} .
$$

Now we are in a position to state the theorem.

THEOREM 2. (H1) Let $\kappa$ be an analytic arc of the type described in $\S 2$ for which the determinant of the $(n-1) \times(n-1)$ matrix

$$
\Delta_{4}(z)=\left\|D_{z}^{2}[G(c)-G(z)]^{j}\right\| \neq 0, \quad 1 \leqq \nu \leqq n-1,1 \leqq j \leqq n-1
$$

for $z$ on $\Omega \cup \kappa \cup \hat{\Omega}$ (arc condition).

$$
\operatorname{det}\left(\begin{array}{cccc}
1 & 0 & \cdots & 0 \\
& a_{j}^{(1)}(0) \\
& \vdots \\
& a_{j}^{(n-1)}(0)
\end{array}\right) \neq 0
$$

where $a_{j}$ are those of Lemma 3 (differential equation condition).

(H3) Let $u=\left(u_{1}, u_{2}, \cdots, u_{n}\right)^{T} \in C^{k}(\Omega \cup \kappa) \cap C^{2}(\Omega),(n \leqq k \leqq 2 n-1, n \geqq 2)$ and satisfy in $\Omega$

$$
u_{x x}+u_{y y}+E u=0
$$

where $E$ is a constant $n \times n$ matrix for which 


$$
\Delta_{1} \equiv\left|\begin{array}{lll}
1, & 0, & \cdots, 0 \\
(E)_{11}, & (E)_{12}, & \cdots,(E)_{1 n} \\
\left(E^{2}\right)_{11}, & \left(E^{2}\right)_{12}, & \cdots,\left(E^{2}\right)_{1 n} \\
\vdots & & \\
\left(E^{n-1}\right)_{11}, & \left(E^{n-1}\right)_{12}, \cdots,\left(E^{n-1}\right)_{1 n}
\end{array}\right| \neq 0 \quad \text { (differential }
$$

where $\left(E^{k}\right)_{i j}$ is the ij component of the $k^{\text {th }}$ power of $E$.

(H4) Let $u$ satisfy on the analytic arc $\kappa$ the boundary conditions

$$
p_{\alpha 1}\left(z, D_{x}, D_{y}\right) u_{1}=f_{\alpha}(z), \quad \alpha=1,2, \cdots, n,
$$

where

$$
p_{\alpha 1}(z, \xi, \eta)=\sum_{r+s \leqq k<2 n} p_{\alpha 1}^{r s}(z) \xi^{r} \eta^{s},
$$

with $p_{\alpha 1}^{r s}(z)$ and $f_{\alpha}(z)$ analytic in $\Omega \cup \kappa \cup \hat{\Omega}$. Moreover if $P_{\alpha 1}(z, \xi, \eta)$ is the principal part of $p_{\alpha 1}(z, \xi, \eta)$ (as polynomial in $\xi$ and $\eta$ ), we assume for

$$
\Delta_{2}(z, \xi, \eta)=\left|\begin{array}{cc}
D_{\xi}^{n-1} P_{11}(z, \xi, \eta), D_{\xi}^{n-2} D_{\eta} P_{11}, \cdots, D_{\eta}^{n-1} P_{11} \\
D_{\xi}^{n-1} P_{12}( & ), D^{n-2} D_{\eta} P_{21}, \cdots, D_{\eta}^{n-1} P_{21} \\
\vdots & \\
D_{\xi}^{n-1} P_{n 1}( & ), D_{\xi}^{n-2} D_{\eta} P_{n 1}, \cdots, D_{\eta}^{n-1} P_{n 1}
\end{array}\right|
$$

that for $z \in \Omega \cup \kappa \cup \Omega$

$$
\Delta_{2}=\Delta_{2}(z, \xi, \eta)_{\xi=1, \eta=i} \neq 0^{2}
$$

and

$$
\Delta_{3}=\Delta_{2}(z, \xi, \eta)_{\xi=1, \eta=-i} \neq 0^{2} .
$$

Then $u=\left(u_{1}, u_{2}, \cdots, u_{n}\right)^{T}$ can be reflected across the boundary conditions (4.15) into $\Omega \cup \kappa \cup \hat{\Omega}$.

Before proceeding to the proof of Theorem 2, we shall state Theorem 3, which deals with the case $k=n-1$, since the proof of Theorem 3 follows the same lines as the proof of Theorem 2. Only in the proof of Theorem 3, Lemmas $4,5,4 \mathrm{~A}$ and $5 \mathrm{~A}$ are unnecessary.

THEOREM 3. Let $\kappa$ be an analytic arc of the type described in $\S 2$. $\left(\mathrm{H} 1^{*}\right) \quad$ Let $u=\left(u_{1}, u_{2}, \cdots, u_{n}\right)^{T} \in C^{n-1}(\Omega \cup \kappa) \cap C^{2}(\Omega)$ and satisfy in $\Omega$

2 These, as Professor Jean Leray has kindly pointed out to me, are conditions on the behavior of the boundary conditions in the characteristic direction. He has also proved that Conditions (H1) and (H2) are always satisfied, i.e. they are unnecessary restrictions. 


$$
u_{x x}+u_{y y}+E u=0
$$

where $E$ is a constant $n \times n$ matrix for which

$$
\Delta_{1} \neq 0
$$

(see Th. 2) .

(H2*) Let $u$ satisfy on the analytic arc $\kappa$ the boundary conditions

$$
p_{\nu}(D) u_{1}=\sum_{r+s \leq n-1} p_{\nu 1}^{r s}(z) D_{x}^{r} D_{y}^{s} u_{1}=f_{\nu}(z), \quad \nu=1,2, \cdots, n,
$$

where $p_{\nu 1}^{r s}(z)$ and $f_{\nu}(z)$ are analytic in $\Omega \cup \kappa \cup \hat{\Omega}$. Also assume in $\Omega \cup \kappa \cup \widehat{\Omega}$

$$
\begin{aligned}
& \Delta_{2}(z, \xi, \eta)_{\xi=1, \eta=i} \neq 0 \\
& \Delta_{2}(z, \xi, \eta)_{\xi=1, \eta=-i} \neq 0
\end{aligned}
$$$$
\text { (see Th. } 2 \text { with } k=n-1 \text { ). }
$$$$
\text { (see Th. } 2 \text { with } k=n-1 \text { ). }
$$

Then $u=\left(u_{1}, u_{2}, \cdots, u_{n}\right)^{T}$ can be reflected across the boundary conditions (4.15*) into $\Omega \cup \kappa \cup \hat{\Omega}$. Moreover the reflection can be reduced to quadratures.

Proof of Theorem 2. We first consider (3.16) with $A^{*}=B^{*}=0$ and

$$
g(\widehat{z})=W^{*}(c, \hat{z}), \quad h(z)=W^{*}(z, c)
$$

and get:

$$
\begin{aligned}
U^{*}(z, \hat{z})= & h(z)+g(\hat{z})-R[z, \hat{z} ; c, c] h(c) \\
& -\int_{c}^{z} R_{t}[z, \hat{z} ; t, c] h(t) d t-\int_{c}^{\hat{z}} R_{\sigma}[z, \hat{z} ; c, \sigma] g(\sigma) d \sigma
\end{aligned}
$$

where, since it was shown that $W^{*}(z, \zeta)$ is an analytic function of $z$ for $z$ in $\Omega$ and an analytic function of $\zeta$ for $\zeta$ in $\hat{\Omega}$, then $h(z)$ is an analytic function of $z$ for $z$ in $\Omega$ and $g(\zeta)$ is an analytic function of $\zeta$ for $\zeta$ in $\hat{\Omega}$. From (3.13) and (3.14) and (H.3) we see that $h(z) \in C^{k}(\Omega \cup \kappa)$ and $g(z) \in C^{k}(\hat{\Omega} \cup \kappa)$.

With the aid of Lemma 3 we get

$$
\begin{aligned}
& R_{t}[z, \hat{z} ; t, c]=\sum_{j=0}^{n-1}[G(c)-G(\hat{z})] a_{j}^{(1)}\{(t-z)[G(c)-G(\widehat{z})]\} E^{j} \\
& R_{o}[z, \hat{z} ; c, \sigma]=\sum_{j=0}^{n-1} G^{\prime}(\sigma)(c-z) a_{j}^{(1)}\{(c-z)[G(\sigma)-G(\widehat{z})]\} E^{j} .
\end{aligned}
$$

Let

$$
\left(e_{m}^{j}, h\right)=e_{m 1}^{j} h_{1}+e_{m 2}^{j} h_{2}+\cdots+e_{m n}^{j} h_{n} .
$$

Then the first component of (4.18) becomes 


$$
U_{1}^{*}(z, \hat{z})=h_{1}^{*}(z, \hat{z})+g_{1}^{*}(z, \hat{z})-\{R[z, \widehat{z} ; c, c] h(c)\}_{1 \text { st component }}
$$

where

$$
h_{1}^{*}(z, \zeta)=h_{1}(z)-G_{1}(\zeta) \sum_{j=0}^{n-1} \int_{c}^{z} a_{j}^{(1)}\left\{(t-z) G_{1}(\zeta)\right\}\left(e_{1}^{j}, h(t)\right) d t
$$

with

$$
G_{1}(\zeta)=G(c)-G(\zeta)
$$

and

$$
\begin{aligned}
g_{1}^{*}(z, \zeta)=g_{1}(\zeta) & -(c-z) \sum_{j=0}^{n-1} \int_{c}^{\zeta} a_{j}^{(1}\{(c-z)[G(\sigma)-G(\zeta)]\} \\
& \times G^{\prime}(\sigma)\left(e_{1}^{j}, g(\sigma)\right) d \sigma .
\end{aligned}
$$

Note that $h_{1}^{*}(z, \zeta)$ is analytic for $(z, \zeta)$ on $\Omega \times \Omega$ and $\in C^{k}[(\Omega \cup \kappa) \times(\Omega \cup \kappa)]$ and that $g_{1}^{*}(z, \zeta)$ is analytic for $(z, \zeta)$ on $\hat{\Omega} \times \hat{\Omega}$ and $\in C^{k}[(\hat{\Omega} \cup \kappa) \times(\hat{\Omega} \cup \kappa)]$.

With the aid of Lemma 1 , the boundary conditions can be written:

$$
\begin{aligned}
f_{\nu}(z)= & \sum_{r+s=k} p_{\nu 1}^{r s}(z)(i)^{s}\left\{\sum_{m=0}^{k} \alpha_{m}^{r s}\left[G^{\prime}(\widehat{z})\right]^{-m} D_{z}^{k-m} D_{\hat{z}}^{m}\right\} \cdot U_{1}^{*}(z, \widehat{z}) \\
& + \text { terms of order } l+m \leqq k-1 \text { in } D_{z}^{l} D_{\hat{z}}^{m} U_{1}^{*} .
\end{aligned}
$$

Apply the boundary conditions to (4.21), evaluate on the boundary $\kappa$, remembering that on $\kappa, z=\widehat{z}$, and substitute the new functions $\widetilde{h}_{j}(z, z)$ and $\widetilde{g}_{j}(z, z),(0 \leqq j \leqq 2 n-1)$ where

$$
\tilde{h}_{j}(z, \zeta)=D_{\zeta}^{j} h_{1}^{*}(z, \zeta), \quad \widetilde{g}_{j}(z, \zeta)=D_{z}^{j} g_{1}^{*}(z, \zeta)
$$

with $D^{\circ} f=f, D^{\circ} g=g$. Thus the boundary conditions become, since $k \geqq n-1$, for $z$ on $\kappa$

$$
\begin{aligned}
& \tilde{f}_{\nu}(z)=\left.\sum_{r+s=k} p_{\nu 1}^{r s}(z)(i)^{s}\left\{\sum_{m=0}^{n-1}+\sum_{m=n}^{k}\right\} \alpha_{m}^{r s}\left[G^{\prime}(z)\right]^{-m} D_{z}^{k-m} \widetilde{h}_{m}(z, \zeta)\right]_{\zeta=z} \\
&+ \text { terms of order } l+m \leqq k-1 \text { in } D_{z}^{l} \widetilde{h}_{m}(z, z) \\
&\left.+\sum_{r+s=k} p_{\nu 1}^{r s}(z)(i)^{s}\left\{\sum_{m=0}^{n-1}+\sum_{m=n}^{k}\right\} \alpha_{k-m}^{r s}\left[G^{\prime}(z)\right]^{m-k} D_{\zeta}^{k-m} \widetilde{g}_{m}(z, \zeta)\right]_{\zeta=z} \\
&+ \text { terms of order } l+m \leqq k-1 \text { in } D_{\zeta}^{l} \widetilde{g}_{m}(z, z) \\
& \nu=1,2, \cdots, n
\end{aligned}
$$

( $\Sigma^{\prime}$ indicates we sum when $k \geqq n$ ),

where $\tilde{f}_{\nu}(z)$ is known and analytic in $\Omega \cup \kappa \cup \hat{\Omega}$. It should be remembered that the first two terms (involving only $\widetilde{h}_{m}$ and their derivatives) are analytic functions of $z$ for $z$ in $\Omega$ and that the last two terms (involving only $\widetilde{g}_{m}$ and their derivatives) are analytic functions of $z$ for $z$ in $\widehat{\Omega}$

Rearranging terms in (4.26) yields for $z$ on $\kappa$ : 


$$
\begin{aligned}
\left.\sum_{m=0}^{n-1} \beta_{\nu m}^{k}(z)\left[G^{\prime}(z)\right]^{-m} D_{z}^{k-m} \widetilde{h}_{m}(z, \zeta)\right]_{\zeta=z} & \\
& \left.\quad+\sum_{m=0}^{n-1} \beta_{\nu, k-m}^{k}(z)\left[G^{\prime}(z)\right]^{m-k} D_{\zeta}^{k-m} \widetilde{g}_{m}(z, \zeta)\right]_{\zeta=z} \\
= & A_{\nu}(z)+\hat{A}_{\nu}(z)+\widetilde{f}_{\nu}(z)
\end{aligned}
$$

where

$$
\begin{aligned}
A_{\nu}(z)= & \left.-\sum_{m=n}^{k \geq n} \beta_{\nu m}^{k}(z)\left[G^{\prime}(z)\right]^{-m} D_{z}^{k-m} \tilde{h}_{m}(z, \zeta)\right]_{\zeta=z} \\
& \left.+ \text { terms of order } l+m \leqq k-1 \text { in } D_{z}^{l} \widetilde{h}_{m}(z, \zeta)\right]_{\zeta=z}
\end{aligned}
$$
with coefficients analytic in $Q \cup \kappa \cup \hat{\Omega}$.

$$
\begin{aligned}
\hat{A}_{\nu}(z)= & \left.-\sum_{m=n}^{k \geq n} \beta_{\nu, k-m}^{k}(z)\left[G^{\prime}(z)\right]^{m-k} D_{\zeta}^{k-m} \widetilde{g}_{m}(z, \zeta)\right]_{\zeta=z} \\
& \left.+ \text { terms of order } l+m \leqq k-1 \text { in } D_{\zeta}^{l} \widetilde{g}_{m}(z, \zeta)\right]_{\zeta=z}
\end{aligned}
$$
with coefficients analytic in $\Omega \cup \kappa \cup \hat{\Omega}$.

$$
\beta_{\nu \mu}^{k}(z)=\sum_{r+s=k} p_{\nu 1}^{r s}(z)(i)^{s} \alpha_{\mu^{r}}^{r s}
$$

with $A_{\nu}(z)$ analytic in $\Omega, \hat{A}_{\nu}(z)$ analytic in $\hat{\Omega}$, and $\beta_{\nu m}^{k}(z)$ and $\tilde{f}_{\nu}(z)$ analytic in $\Omega \cup \kappa \cup \hat{\Omega}$.

Our next goal is to convert (4.27) into a system of Volterra integral equations for the $n$ functions $D_{z}^{k-m} \widetilde{h}_{m}(z, z), 0 \leqq m \leqq n-1$. On $\kappa$, the system is to be satisfied and we shall see that they also have an analytic solution for $z$ in $\hat{\Omega} \cup \kappa$. With this in mind we state and prove two lemmas.

\section{LEMMA 4. Let $k \geqq n$}

$$
\left.\left.\alpha_{k-m}(z)=D_{z}^{k-m} D_{\zeta}^{m} h_{1}^{*}(z, \zeta)\right]_{\zeta=z}=D_{z}^{k-m} \tilde{h}_{m}(z, \zeta)\right]_{\zeta=z}, \quad 0 \leqq m \leqq k
$$

and the hypotheses $(\mathrm{H} 1),(\mathrm{H} 2)$, and $(\mathrm{H} 3)$ of the theorem hold. Then

$$
\alpha_{k-m}(z)=\sum_{r=0}^{n-1} \int_{c}^{z} K_{k-m, r}(t, z) \alpha_{k-r}(t) d t+C_{k-m}(z), \quad n \leqq m \leqq k
$$

where $K_{k-m, r}(t, z)$ are analytic for $t$ and $z$ in $\Omega \cup \kappa \cup \hat{\Omega}$ and $C_{k-m}(z)$ is analytic for $z$ in $\Omega \cup \kappa \cup \hat{\Omega}$.

Proof. Since the $a_{j}(\sigma)$ occuring in (4.22) are entire functions, the following Taylor's expansion with remainder is valid:

$$
a_{j}^{(1)}\left[(t-z) G_{1}\right]=p_{n-2}(t-z)+R_{j, n-2}(t, z)
$$


where $p_{n-2}(\sigma)$ is a polynomial of degree $n-2$ in $\sigma$ and

$$
R_{j, n-2}(t, z)=\frac{G_{1}^{n-1}}{(n-2) !} \int_{z}^{t}(t-\sigma)^{n-2} a_{j}^{(n)}\left[(\sigma-z) G_{1}\right] d \sigma .
$$

Introducing this into (4.22) and interchanging the order of integration for the remainder yields:

$$
\begin{aligned}
h_{1}^{*}(z, \zeta)=h_{1}(z) & -G_{1}(\zeta) \sum_{j=0}^{n-1} a_{j}^{(1)}(0) \int_{c}^{z}\left(e_{1}^{j}, h(t)\right) d t \\
& -G_{1}^{2}(\zeta) \sum_{j=0}^{n-1} a_{j}^{(2)}(0) \int_{c}^{z}(t-z)\left(e_{1}^{j}, h(t)\right) d t \\
& -\frac{1}{2 !} G_{1}^{3}(\zeta) \sum_{j=0}^{n-1} a_{j}^{(3)}(0) \int_{c}^{z}(t-z)^{2}\left(e_{1}^{j}, h(t)\right) d t-\cdots \\
& -\frac{1}{(n-2) !} G_{1}^{n-1}(\zeta) \sum_{j=0}^{n-1} a_{j}^{(n-1)}(0) \int_{c}^{z}(t-z)^{n-2}\left(e_{1}^{j}, h(t)\right) d t \\
& +\frac{1}{(n-2) !} G_{1}^{n}(\zeta) \sum_{j=0}^{n-1} \int_{c}^{z} a_{j}^{(n)}\left[(s-z) G_{1}(\zeta)\right] \\
& \cdot\left(\int_{c}^{s}(\sigma-s)^{n-2}\left(e_{1}^{j}, h(\sigma)\right) d \sigma\right) d s .
\end{aligned}
$$

Next let

$$
\begin{array}{ll}
B_{r}(z)=\frac{1}{(r-1) !} \sum_{j=0}^{n-1} a_{j}^{(r)}(0)\left(e_{1}^{j}, h(z)\right), & 1 \leqq r \leqq n-1 \\
B_{0}(z)=h_{1}(z) . &
\end{array}
$$

Then, since by assumption

$$
\operatorname{det}\left(\begin{array}{cc}
1 & 0 \cdots \cdots \cdot 0 \\
& a_{j}^{(1)}(0) \\
\vdots \\
a_{j}^{(n-1)}(0)
\end{array}\right) \neq 0, \quad 0 \leqq j \leqq n-1
$$

we can invert the system of equations and get

$$
\left(e_{1}^{j}, h(z)\right)=\sum_{r=0}^{n-1} b_{j r} B_{r}(z)
$$

where $\left(b_{j r}\right)$ is a constant matrix. Thus the expression for $h_{1}^{*}$ can be written: 


$$
\begin{aligned}
h_{1}^{*}(z, \zeta)=B_{0}(z) & -G_{1}(\zeta) \int_{c}^{z} B_{1}(t) d t \\
& -G_{1}^{2}(\zeta) \int_{c}^{z}(t-z) B_{2}(t) d t-\cdots \\
& -G_{1}^{n-1}(\zeta) \int_{c}^{z}(t-z)^{n-2} B_{n-1}(t) d t \\
& +\frac{1}{(n-2) !} G_{1}^{n}(\zeta) \sum_{j=0}^{n-1} \int_{c}^{z} a_{j}^{(n)}\left[(s-z) G_{1}(\zeta)\right] \\
& \cdot\left(\int_{c}^{s}(\sigma-s)^{n-2} \sum_{r=0}^{n-1} b_{j r} B_{r}(\sigma) d \sigma\right) d s .
\end{aligned}
$$

Next we let

$$
\begin{aligned}
& F_{0}(z)=B_{0}(z) \\
& F_{r}(z)=-\int_{c}^{z}(t-z)^{r-1} B_{r}(t) d t
\end{aligned}
$$

and thus

$$
F_{r}^{(r)}(z)=(-1)^{r}(r-1) ! B_{r}(z) .
$$

Introducing these into (4.32) gives

$$
\begin{aligned}
h_{1}^{*}(z, \zeta)=F_{0}(z)+ & G_{1}(\zeta) F_{1}(z)+G_{1}^{2}(\zeta) F_{2}(z)+\cdots+G_{1}^{n-1}(\zeta) F_{n-1}(z) \\
+ & \frac{1}{(n-2) !} G_{1}^{n}(\zeta) \sum_{j=0}^{n-1} \sum_{r=0}^{n-1} b_{j r} \frac{(-1)^{r}}{(r-1) !} \int_{c}^{z} a_{j}^{(n)}\left[(s-z) G_{1}(\zeta)\right] \\
& \cdot\left(\int_{c}^{s}(\sigma-s)^{n-2} F_{r}^{(r)}(\sigma) d \sigma\right) d s .
\end{aligned}
$$

Consider now for $0 \leqq m \leqq k$

$$
\begin{aligned}
D_{z}^{k-m} & \left.D_{\zeta}^{m} h_{1}^{*}(z, \zeta)\right]_{\zeta=z}=\delta_{m 0} F_{0}^{(k-m)}(z)+D_{z}^{m}\left[G_{1}(z)\right] F_{1}^{(k-m)}(z) \\
+ & D_{z}^{m}\left[G_{1}^{2}(z)\right] F_{2}^{(k-m)}(z)+\cdots+D_{z}^{m}\left[G_{1}^{n-1}(z)\right] F_{n-1}^{(k-m)}(z) \\
+ & \frac{1}{(n-2) !} \sum_{j=0}^{n-1} \sum_{r=0}^{n-1} b_{j r} \frac{(-1)^{r}}{(r-1) !} D_{z}^{k-m} \int_{c}^{z} D_{\zeta}^{m}\left\{G_{1}^{n}(\zeta)\right. \\
& \left.\left.\cdot a_{j}^{(n)}\left[(s-z) G_{1}(\zeta)\right]\right\}\left(\int_{c}^{s} F_{r}^{(r)}(\sigma)(\sigma-s)^{n-2} d \sigma\right) d s\right]_{\zeta=z},
\end{aligned}
$$

where $\delta_{m 0}$ is the Kronecker delta. Since

$$
\begin{aligned}
k-m+r-(n-2)-2 & \leqq k-m+(n-1)-(n-2)-2 \\
& =k-m-1<k-m, \\
k-m-r+(n-2)+1 & \geqq k-m-(n-1)+(n-2)+1=k-m,
\end{aligned}
$$

the last term involving the integrals is of the form: 


$$
\sum_{r=0}^{n-1} \int_{c}^{z} K_{r}(t, z) F_{r}^{(k-m)}(t) d t+\widetilde{C}_{k-m}(z)
$$

where the $K_{r}(t, z)$ are analytic for $t$ and $z$ in $\Omega \cup \kappa \cup \hat{\Omega}$ and $\widetilde{C}_{k-m}(z)$ is analytic for $z$ in $\Omega \cup \kappa \cup \hat{\Omega}$. This follows since $r \leqq n-1$ and 1 .

$$
\begin{aligned}
& \frac{1}{(n-2) !} \int_{c}^{s} F_{r}^{(r)}(\sigma)(\sigma-s)^{n-2} d \sigma \\
& \quad=\frac{(-1)^{r-k+m}}{[k-m-r+n-2] !} \int_{c}^{s} F_{r}^{(k-m)}(\sigma)(\sigma-s)^{k-m-r+n-2} d \sigma+C_{r}^{*}(s)
\end{aligned}
$$

if $k-m>0$ or, if $k-m=0$ and $r<n-1$, where $C_{r}^{*}(s)$ is a polynomial in $s$. The only difference in the case $k-m=0, r=n-1$ is that the integral on the right side is replaced by $F_{n-1}(s)$. And since 2 .

$$
\int_{c}^{z} K^{*}(s, z)\left(\int_{c}^{s} F_{r}^{(k-m)}(\sigma)(\sigma-s)^{l} d \sigma\right) d s=\int_{c}^{z} K^{* *}(s, z) F_{r}^{(k-m)}(\sigma) d \sigma
$$

where $K^{* *}(s, z)$ is an analytic function of $s$ and $z$ (since $K^{*}(s, z)$ is) for $s$ and $z$ in $\Omega \cup \kappa \cup \hat{\Omega}$. The last integral follows from integration by parts $l+1$ times.

Thus by the definition of $\alpha_{k-m}(z),(4.30),(4.33)$, and (4.34) we get for $0 \leqq m \leqq k$

$$
\begin{aligned}
\alpha_{k-m}(z)= & \delta_{m 0} F_{0}^{(k-m)}(z)+D_{z}^{m}\left[G_{1}(z)\right] F_{1}^{(k-m)}(z) \\
& +D_{z}^{m}\left[G_{1}^{2}(z)\right] F_{2}^{(k-m)}(z)+\cdots+D_{z}^{m}\left[G_{1}^{n-1}(z)\right] F_{n-1}^{(k-m)}(z) \\
& +\sum_{r=0}^{n-1} \int_{c}^{z} \widetilde{L}_{r, k-m}(t, z) F_{r}^{(k-m)}(t) d t \\
& +\widetilde{C}_{k-m}(z)
\end{aligned}
$$

where for $t, z \in \Omega \cup \kappa \cup \hat{\Omega}, \widetilde{L}_{r, k-m}(t, z)$ and $C_{k-m}(z)$ are analytic.

Next we consider for $0 \leqq m \leqq n-2<k$

$$
\begin{aligned}
A_{k-m}^{*}(z) \equiv & \frac{1}{(n-m-2) !} \int_{c}^{z}(z-t)^{n-m-2} \alpha_{k-m}(t) d t \\
= & \delta_{m 0} F_{0}^{(k-(n-1))}(z)+D_{z}^{m}\left[G_{1}(z)\right] F_{1}^{(k-(n-1))}(z) \\
& +D_{z}^{m}\left[G_{1}^{2}(z)\right] F_{2}^{(k-(n-1))}(z)+\cdots \\
& +D_{z}^{m}\left[G_{1}^{n-1}(z)\right] F_{n-1}^{(k-(n-1))}(z) \\
& +\sum_{r=0}^{n-1} \int_{c}^{z} L_{k-m, r}(t, z) F_{r}^{(k-(n-1))}(t) d t+R_{k-m}(z)
\end{aligned}
$$

where we have integrated by parts $n-m-1$ times. $R_{k-m}(z)$ and $L_{k-m, r}$ are functions analytic for $z, t$ in $\Omega \cup \kappa \cup \hat{\Omega}$. For consistency let $A_{k-(n-1)}^{*}(z)=\alpha_{k-(n-1)}(z)$ and $R_{k-(n-1)}=\widetilde{C}_{k-(n-1)}(z), L_{k-(n-1), r}=L_{r, k-(n-1)}$.

Since by hypothesis we have for $z$ in $\Omega \cup \kappa \cup \hat{\Omega}$ 


$$
\left|\Delta_{4}(z)\right|=\left|\begin{array}{cc}
1 & G_{1} \cdots G_{1}^{n-1} \\
0 & \\
\vdots & D_{z}^{m} G_{1}^{\nu}(z) \\
0 &
\end{array}\right| \neq 0, \quad 1 \leqq m \leqq n-1,1 \leqq \nu \leqq n-1
$$

the above system, coupled with $\alpha_{k-(n-1)}(z)$ in (4.35) can be written as:

$$
\begin{aligned}
F^{(k-(n-1))}(z)=\Delta_{4}^{-1}(z) A^{*}(z) & -\Delta_{4}^{-1}(z) \int_{c}^{z} L(t, z) F^{(k-(n-1))}(t) d t \\
& -\Delta_{4}^{-1}(z) R(z)
\end{aligned}
$$

with:

$$
\begin{gathered}
F^{(k-(n-1))}(z)=\left(\begin{array}{c}
F_{0}^{(k-(n-1))}(z) \\
F_{1}^{(k-(n-1))}(z) \\
\vdots \\
F_{n-1}^{(k-(n-1))}(z)
\end{array}\right), \\
A^{*}(z)=\left(\begin{array}{c}
A_{k}^{*}(z) \\
A_{k-1}^{*}(z) \\
\vdots \\
A_{k-(n-1)}^{*}(z)
\end{array}\right)=\left(\begin{array}{c}
\frac{1}{(n-2) !} \int_{c}^{z}(z-t)^{n-2} \alpha_{k}(t) d t \\
\frac{1}{(n-3) !} \int_{c}^{z}(z-t)^{n-3} \alpha_{k-1}(t) d t \\
\vdots \\
\left\{\begin{array}{c}
(n-1) \\
k-(n-1) \leqq k-m
\end{array}\right) \\
k(t, z)=\left(L_{k-m, r}(t, z)\right) \\
R(z)=\left(R_{k}(z), R_{k-1}(z), \cdots, R_{k-(n-1)}(z)\right)^{T} \cdot
\end{array}\right.
\end{gathered}
$$

We consider (4.37) as a system of Volterra integral equations and obtain its solution in the form:

$$
\begin{aligned}
F^{(k-(n-1))}(z)= & \Delta_{4}^{-1}(z)\left[A^{*}(z)-R(z)\right] \\
& -\Delta_{4}^{-1}(z) \int_{c}^{z} \Gamma(t, z) \Delta_{4}^{-1}(t)\left[A^{*}(t)-R(t)\right] d t
\end{aligned}
$$

where $\Gamma(t, z)$ is the resolvent matrix which is an analytic function of $t$ and $z$ for $t$ and $z$ in $\Omega \cup \kappa \cup \hat{\Omega}$.

Now we are in a position to express $\alpha_{k-m}(z), n \leqq m \leqq k$ in terms of $\alpha_{k-m}(z), 0 \leqq m \leqq n-1$. To this end we consider (4.35), which is valid for $0 \leqq m \leqq k$ and combine with it the integrated expression of (4.38): 


$$
F^{(k-m)}(z)=\int_{c}^{z} \Gamma_{k-m}^{*}(t, z) A^{*}(t) d t+R^{*}(z), \quad n \leqq m \leqq k
$$

where $\Gamma_{k-m}^{*}(t, z)$ is analytic for $t$ and $z$ in $\Omega \cup \kappa \cup \hat{\Omega}$ and $R^{*}(z)$ is analytic for $z$ in $\Omega \cup \kappa \cup \hat{\Omega}$. This combination gives:

$$
\alpha_{k-m}(z)=\sum_{r=0}^{n-1} \int_{c}^{z} \Gamma_{r, k-m}^{* *}(t, z) A_{k-r}^{*}(t) d t+R_{k-m}^{* *}(z), \quad n \leqq m \leqq k
$$

where $R_{k-m}^{* *}(z)$ is analytic for $z$ in $\Omega \cup \kappa \cup \hat{\Omega}$ and $\Gamma_{r, k-m}^{* *}(t, z)$ is analytic for $t$ and $z$ in $\Omega \cup \kappa \cup \hat{\Omega}$. But from the definition of $A_{k-r}^{*}(z)$ in (4.36) for $0 \leqq r \leqq n-2$ and $A_{k-(n-1)}^{*}(z)=\alpha_{k-(n-1)}(z)$, we get the result upon integrating (4.39) by parts if necessary.

Thus Lemma 4 is proved.

With the notation and assumptions of Lemma 4, we next state and prove:

Lemma 5. For $l+m \leqq k-1$

$$
\left.D_{z}^{\imath} \widetilde{h}_{m}(z, \zeta)\right]_{\zeta=z}=\sum_{j=0}^{n-1} \int_{c}^{z} \widetilde{K}_{k-m, j}^{*}(t, z) \alpha_{k-j}(t) d t+\widetilde{C}_{k-m}^{*}(z)
$$

where $\widetilde{K}_{k-m, j}^{*}(t, z)$ are analytic for $t$ and $z$ in $\Omega \cup \kappa \cup \hat{\Omega}$ and $C_{k-m}^{*}(z)$ are analytic for $z$ in $\Omega \cup \kappa \cup \hat{\Omega}$.

Proof. In the notation of Lemma 4 , since $l+m \leqq k-1$

$$
\begin{aligned}
D_{t}^{(k-l-m)}\left[D_{z}^{\imath} \tilde{h}_{m}(z, \zeta)\right]_{z=\zeta=t} & \left.=\sum_{j=0}^{k-l-m}\left(\begin{array}{c}
k-l-m \\
j
\end{array}\right) D_{\zeta}^{j} D_{z}^{k-l-m-j} D_{z}^{\imath} \tilde{h}_{m}(z, \zeta)\right]_{z=\zeta=t} \\
& \left.=\sum_{j=0}^{k-l-m}\left(\begin{array}{c}
k-l-m \\
j
\end{array}\right) D_{z}^{k-m-j} \tilde{h}_{m+j}(z, \zeta)\right]_{z=\zeta=t} \\
& =\sum_{j=0}^{k-l-m}\left(\begin{array}{c}
k-l-m \\
j
\end{array}\right) \alpha_{k-m-j}(t) .
\end{aligned}
$$

Thus

$$
\begin{aligned}
D_{z}^{l} \widetilde{h}_{m}(z, \zeta)_{z=\zeta=t}= & \frac{1}{(k-l-m-1) !} \int_{c}^{t}(t-s)^{k-l-m-1} \\
& \cdot\left[\sum_{j=0}^{k-l-m}\left(\begin{array}{c}
k-l-m \\
j
\end{array}\right) \alpha_{k-m-j}(s)\right] d s \\
& + \text { polynomial in }(t-c) \\
= & \sum_{j=0}^{n-1} \int_{c}^{t} \widetilde{K}_{k-m, j}^{*}(s, t) \alpha_{k-j}(s) d t+\widetilde{C}_{k-m}^{*}(t)
\end{aligned}
$$

with $\widetilde{C}_{k-m}^{*}(z)$ analytic for $z$ in $\Omega \cup \kappa \cup \hat{\Omega}, \widetilde{K}_{k-m, j}^{*}(t, z)$ is analytic for $t$ and $z$ in $Q \cup \kappa \cup \hat{\Omega}$ and where we have made use of Lemma 4 and the fact that if $r \geqq 0$ then 


$$
\int_{c}^{z} d t(z-t)^{r} \int_{c}^{t} K(s, t) \alpha_{k-j}(s) d s=\frac{1}{r+1} \int_{c}^{z}(z-t)^{r+1} K(t, t) \alpha_{k-j}(t) d t
$$

for the case when $m+j \geqq n$. Thus the Lemma is proved.

We are now in a position to continue the proof of the theorem. Combining (4.28) with the results (4.31) of Lemma 4 yields:

$$
\begin{aligned}
A_{\nu}(z)= & \sum_{j=0}^{n-1} \int_{c}^{z} K_{\nu j}^{* *}(t, z) \alpha_{k-j}(t) d t \\
& + \text { terms of order } l+m \leqq k-1 \text { in } \\
& D_{z}^{\iota} \tilde{h}_{m}(z, \zeta)_{\zeta=z} \text { with coefficients analytic in } \Omega \cup \kappa \cup \hat{\Omega},
\end{aligned}
$$

where $K_{\nu j}^{* *}(t, z)$ are analytic for $t$ and $z$ in $\Omega \cup \kappa \cup \hat{\Omega}$. With the aid of Lemma 5 applied to the second term on the right we get:

$$
A_{\nu}(z)=\sum_{m=0}^{n-1} \int_{c}^{z} \widetilde{K}_{\nu m}(t, z) \alpha_{k-m}(t) d t+\widetilde{C}_{\nu}(z)
$$

where $\widetilde{K}_{\nu j}(t, z)$ are analytic in $t$ and $z$ for $t$ and $z$ in $\Omega \cup \kappa \cup \hat{\Omega}$, and $\widetilde{C}_{\nu}(z)$ is analytic for $z$ in $\Omega \cup \kappa \cup \hat{\Omega}$.

Finally, we combine (4.27) and (4.42) and get for $z$ on $\kappa$ :

$$
\beta_{0}(z) \Phi(z)=\int_{c}^{z} \widetilde{K}(t, z) \Phi(t) d t+g^{*}(z),
$$

where $g^{*}(z)$ is an analytic vector function for $z$ in $\hat{\Omega}$ and in $C(\Omega \cup \kappa)$

$$
\begin{aligned}
\widetilde{K}(t, z)= & \left(\widetilde{K}_{\nu m}(t, z)\right), \\
\Phi(z)= & \left(\alpha_{k}(z), \alpha_{k-1}(z), \cdots, \alpha_{k-n+1}(z)\right)^{T}, \\
\beta_{0}(z)= & \left(\beta_{\nu m}^{k}(z)\left[G^{\prime}(z)\right]^{-m}\right), \\
& m \text { designates the column, } \nu \text { the row. } \\
0 \leqq & m \leqq n-1,1 \leqq \nu \leqq n .
\end{aligned}
$$

But from (4.29) we see that

$$
\left|\beta_{\nu \mu}^{k}(z)\right|=\left|\begin{array}{cc}
\sum_{r+s=k}(i)^{s} \alpha_{0}^{r s} p_{11}^{r s}(z), & \sum_{r+s=k}(i)^{s} \alpha_{1}^{r s} p_{11}^{r s}, \cdots, \sum_{r+s=k}(i)^{s} \alpha_{n-1}^{r s} p_{11}^{r s} \\
\sum_{r+s=k}(i)^{s} \alpha_{0}^{r s} p_{21}^{r s}(z), & \sum_{r+s=k}(i)^{s} \alpha_{1}^{r s} p_{21}^{r s}, \cdots, \sum_{r+s=k}(i)^{s} \alpha_{n-1}^{r s} p_{21}^{r s} \\
\vdots & \\
\sum_{r+s=k}(i)^{s} \alpha_{0}^{r s} p_{n 1}^{r s}(z), & \sum_{r+s=k}(i)^{s} \alpha_{1}^{r s} p_{n 1}^{r s}, \cdots, \sum_{r+s=k}(i)^{s} \alpha_{n-1}^{r s} p_{n 1}^{r s}
\end{array}\right|
$$

where by definition of $\alpha_{\mu}^{r s}$

$$
(a+b)^{r}(a-b)^{s}=\sum_{\mu=0}^{r+s} \alpha_{\mu}^{r s} a^{r+s-\mu} b^{\mu}
$$

and by definition 


$$
P_{\nu 1}(z, \xi, \eta)=\sum_{r+s=k} p_{\nu 1}^{r s}(z) \xi^{r} \eta^{s}
$$

Thus if $\zeta=\xi+i \eta, \bar{\zeta}=\xi-i \eta$ then

$$
P_{\nu 1}(z, \xi, \eta)=2^{-k} \sum_{r+s=k}(i)^{s} p_{\nu 1}^{r s}(z)(\bar{\zeta}+\zeta)^{r}(\bar{\zeta}-\zeta)^{s}
$$

and

$$
\left.D_{\xi}^{j} P_{\nu 1}(z, \xi, \eta)\right|_{\substack{\xi=1 \\ \eta=i}}=j ! 2^{-j} \sum_{r+s=k}(i)^{s} \alpha_{j}^{r s} p_{\nu 1}^{r s}(z)
$$

$$
\begin{aligned}
& \left|\beta_{\nu \mu}^{k}(z)\right|= \\
& \quad=C_{n}\left|\begin{array}{c}
P_{11}(z, \xi, \eta),\left(D_{\xi}-i D_{\eta}\right) P_{11}(z, \xi, \eta), \cdots,\left(D_{\xi}-i D_{\eta}\right)^{n-1} P_{11}(z, \xi, \eta) \\
P_{21}(z, \xi, \eta),\left(D_{\xi}-i D_{\eta}\right) P_{21}(z, \xi, \eta), \cdots,\left(D_{\xi}-i D_{\eta}\right)^{n-1} P_{21}(z, \xi, \eta) \\
\vdots \\
P_{n 1}(z, \xi, \eta),\left(D_{\xi}-i D_{\eta}\right) P_{n 1}(z, \xi, \eta), \cdots,\left(D_{\xi}-i D_{\eta}\right)^{n-1} P_{n 1}(z, \xi, \eta)
\end{array}\right| \begin{array}{l}
\substack{\xi=1 \\
\eta=i} \\
\vdots
\end{array} \mid
\end{aligned}
$$

with $C_{n}=1 ! 2 ! \cdots(n-1)$ !

Since $P_{\nu 1}(z, \xi, \eta)$ are homogeneous polynomials in $(\xi, \eta)$ of degree $k$, we see from Euler's formula that

$$
\left(\xi D_{\xi}+\eta D_{\eta}\right)^{l} P_{\nu 1}(z, \xi, \eta)=k^{l} P_{\nu 1}(z, \xi, \eta) \text {. }
$$

Thus

$$
\left|\beta_{\nu \mu}^{k}(z)\right|=(k)^{\{-n(n-1)\} / 2} C_{n}\left|\left(\xi D_{\xi}+\eta D_{\eta}\right)^{n-l-1}\left(\xi D_{\xi}-\eta D_{\eta}\right)^{l} P_{\nu 1}(z, \xi, \eta)\right|_{\substack{\xi=1 \\ \eta=i}} \neq 0
$$

if and only if $\left.\Delta_{2}(z, \xi, \eta)\right|_{\xi=1, \eta=i} \neq 0$. This follows immediately upon writting the determinant as a sum of determinants. Thus we have

$$
\left|\beta_{0}(z)\right|=\left[G^{\prime}(z)\right]^{[-n(n-1)\} / 2}\left|\beta_{\nu \mu}^{k}(z)\right| \neq 0^{3}
$$

for $z$ in $\Omega \cup \kappa \cup \hat{\Omega}$ by assumption (4.16). Thus

$$
\Phi(z)=\beta_{0}^{-1}(z) \int_{c}^{z} \widetilde{K}(t, z) \Phi(t)+\beta_{0}^{-1}(z) g^{*}(z) .
$$

We now consider (4.43) as a system of Volterra integral equations in $\Phi(z)$ for $z$ in $\hat{\Omega} \cup \kappa$. As such, this system has a unique solution vector $\Phi_{e}(z)$ which is analytic for $z$ in $\hat{\Omega}$ and continuous for $z$ in $\hat{\Omega} \cup \kappa$ and moreover agrees with $\Phi(z)$ for $z$ on $\kappa$. Thus $\Phi_{e}(z)$ furnishes the analytic continuation of $\Phi(z)$ into $\hat{\Omega} \cup \kappa$. Thus $\Phi(z)$ is analytic for $z$ in $\Omega \cup \kappa \cup \hat{\Omega}$. From the definition of $\Phi(z)$ in (4.43.A) we see this yields the analytic continuation of $\alpha_{k}(z), \alpha_{k-1}(z), \cdots, \alpha_{k-n+1}(z)$ into $\Omega \cup \kappa \cup \hat{\Omega}$. But by (4.36), the definition of $A^{*}(z)$ and (4.38) we get the analytic continuation of $F^{(k-(n-1))}(z)$ into $\Omega \cup \kappa \cup \hat{\Omega}$. By integration, we get the analytic continuation of $F(z)$ into $\Omega \cup \kappa \cup \hat{\Omega}$. (We adjust the

\footnotetext{
${ }^{3}$ Professor J. Leray pointed out to me the relation between $\beta_{\nu \mu}^{k}$ and $\Delta_{2}(z, \xi, \eta)_{\substack{\xi=1 \\ \eta=i}}$.
} 
constants of integration to agree with $F(z)$ and its derivatives at the point $c$ of the boundary; this gives uniqueness.)

Upon differentiating $F_{j}(z)$ and using $(4.32 \mathrm{~A})$ and $(4.31 \mathrm{~A})$ we get the analytic continuation of $\left(e_{1}^{j}, h(z)\right)$ into $\Omega \cup \kappa \cup \hat{\Omega}$. However by assumption (4.14)

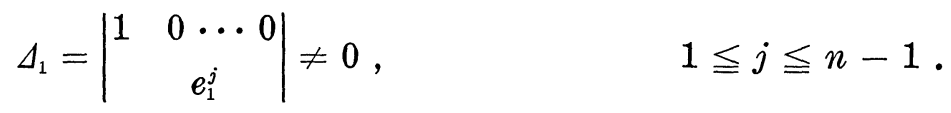

Thus we get the analytic continuation of $h_{1}(z), h_{2}(z), \cdots, h_{n}(z)$ into $\Omega \cup \kappa \cup \hat{\Omega}$.

In a completely analogous way, we can show how to analytically extend $g_{k i}(z), g_{k-1}(z), \cdots, g_{k-(n-1)}(z)$ into $\Omega \cup \kappa \cup \hat{\Omega}$, knowing initially only that they are analytic in $\hat{\Omega}$ and continuous on $\hat{\Omega} \cup \kappa$. In this direction we first note that we have:

LEMMA $4-\mathrm{B}$. Let $k \geqq n$

$$
\alpha_{k-m}^{*}(z)=D_{\zeta}^{k-m} D_{z}^{m} g_{1}^{*}(z, \zeta)=D_{\zeta}^{k-m} \widetilde{g}_{m}(z, z), \quad 0 \leqq m \leqq k
$$

and the hypotheses of the theorem hold. Then

$$
\alpha_{k-m}^{*}(z)=\sum_{r=0}^{n-1} \int_{c}^{z} \hat{K}_{k-m, r}(t, z) \alpha_{k-r}^{*}(t) d t+\hat{C}_{k-m}(z)
$$

where $\hat{K}_{k-m, r}(t, z)$ are analytic for $t$ and $z$ in $\Omega \cup \kappa \cup \hat{\Omega}$ and $\hat{C}_{k-m}(z)$ is analytic for $z$ in $\Omega \cup \kappa \cup \hat{\Omega}$.

Proof. The proof of this lemma is the same as that of Lemma 4 , with only obvious modifications. In place of the expression for the Taylor's expansion for $a_{j}$ about $t=z$ we start with the expression for the Taylor's expansion for $a_{j}$ about $G_{1}(\sigma)=G(\zeta)$ and integrate viz:

$$
\begin{aligned}
g_{1}^{*}(z, \zeta)= & g_{1}(\zeta)-(c-z) \sum_{j=0}^{n-1} a_{j}^{(1)}(0) \int_{c}^{\zeta} G^{\prime}(\sigma)\left(e_{1}^{j}, g(\sigma)\right) d \sigma \\
& -(c-z)^{2} \sum_{j=0}^{n-1} a_{j}^{(2)}(0) \int_{c}^{\zeta}[G(\sigma)-G(\zeta)] G^{\prime}(\sigma)\left(e_{1}^{j}, g(\sigma)\right) d \sigma \\
& -\frac{(c-z)^{3}}{2 !} \sum_{j=0}^{n-1} a_{j}^{(3)}(0) \int_{c}^{\zeta}[G(\sigma)-G(\zeta)]^{2} G^{\prime}(\sigma)\left(e_{1}^{j}, g(\sigma)\right) d \sigma-\cdots \\
& -\frac{1}{(n-2) !}(c-z)^{n} \sum_{j=0}^{n-1} \int_{c}^{\zeta} a_{j}^{(n)}[(G(s)-G(\zeta))(c-z)] \\
& \cdot\left(\int_{c}^{s}[G(\sigma)-G(s)]^{n-2} G^{\prime}(\sigma)\left(e_{1}^{j}, g(\sigma)\right) d \sigma\right) d s .
\end{aligned}
$$

In place of $B_{r}(z)$ we introduce: 


$$
\begin{aligned}
& \widetilde{B}_{r}(\zeta)=\frac{1}{(r-1) !} \sum_{j=0}^{n-1} a_{j}^{(r)}(0)\left(g_{1}^{j}, e(\zeta)\right), \\
& \widetilde{B}_{0}(\zeta)=g_{1}(\zeta)
\end{aligned}
$$

and the expression (4.32) becomes replaced by:

$$
\begin{aligned}
g_{1}^{*}(z, \zeta)=\widetilde{B}_{0}(\zeta) & -(c-z) \int_{c}^{\zeta} \widetilde{B}_{1}(\sigma) G^{\prime}(\sigma) d \sigma \\
& -(c-z)^{2} \int_{c}^{\zeta} \widetilde{B}_{2}(\sigma)[G(\sigma)-G(\zeta)] G^{\prime}(\sigma) d \sigma \\
& -(c-z)^{3} \int_{c}^{\zeta} \widetilde{B}_{3}(\sigma)[G(\sigma)-G(\zeta)]^{2} G^{\prime}(\sigma) d \sigma-\cdots
\end{aligned}
$$

Also $F_{r}(z)$ is replaced by

$$
\widetilde{F}_{r}(\zeta)=-\int_{c}^{\zeta}[G(\sigma)-G(\zeta)]^{r-1} G^{\prime}(\sigma) \widetilde{B}_{r}(\sigma) d \sigma
$$

so

$$
\begin{aligned}
\widetilde{F}_{r}^{(r)}(\zeta)= & (-1)^{r}(r-1) !\left(G^{\prime}(\zeta)\right)^{r} \widetilde{B}_{r}(\zeta) \\
& -\int_{c}^{\zeta} D_{\zeta}^{(r)}[G(\sigma)-G(\zeta)]^{r-1} G^{\prime}(\sigma) \widetilde{B}_{r}(\sigma) d \sigma .
\end{aligned}
$$

Considering these as Volterra integral equations for $\widetilde{B}_{r}(\zeta)$, we can solve, since $G^{\prime}(\zeta) \neq 0$ in $\Omega \cup \kappa \cup \hat{\Omega}$, and get:

$$
\begin{aligned}
\widetilde{B}_{r}(\zeta)= & (-1)^{r} \frac{1}{(r-1) !}\left[G^{\prime}(\zeta)\right]^{-r} \widetilde{F}_{r}^{(r)}(\zeta) \\
& +(-1)^{r} \frac{1}{(r-1) !}\left[G^{\prime}(\zeta)\right]^{-r} \int_{c}^{\zeta} \widetilde{Q}_{r}(\sigma, \zeta) F_{r}^{(r)}(\sigma) d \sigma
\end{aligned}
$$

where $\widetilde{Q}_{r}(\sigma, \zeta)$ is the resolvent which is analytic for $\sigma$ and $\zeta$ in $\Omega \cup \kappa \cup \hat{\Omega}$. Thus

$$
\begin{aligned}
g_{1}^{*}(z, \zeta)=\widetilde{F}_{0}(\zeta)+ & (c-z) \widetilde{F}_{1}(\zeta)+(c-z)^{2} \widetilde{F}_{2}(\zeta) \\
+ & \cdots+(c-z)^{n-1} \widetilde{F}_{n-1}(\zeta) \\
& -\frac{1}{(n-2) !}(c-z)^{n} \sum_{j=0}^{n-1} \sum_{r=0}^{n-1} \frac{(-1)^{r}}{(r-1) !} b_{j r} \\
& \cdot \int_{c}^{\zeta} a_{j}^{(n)}[(G(s)-G(\zeta))(c-z)] \\
& \cdot\left(\int_{c}^{s}[G(\sigma)-G(s)]^{n-2}\left[G^{\prime}(\sigma)\right]^{-r+1}\right. \\
& \left.\cdot\left\{\widetilde{F}_{r}^{(r)}(\sigma)+\int_{c}^{\sigma} \widetilde{Q}_{r}(\tau, \sigma) \widetilde{F}_{r}^{(r)}(\tau) d \tau\right\} d \sigma\right) d s
\end{aligned}
$$

and for $0 \leqq m \leqq n-1$ 


$$
\begin{aligned}
\alpha_{k-m}^{*}(z)= & \left.D_{\zeta}^{k-m} D_{z}^{m} g_{1}^{*}(z, \zeta)\right]_{z=\zeta} \\
= & (-1)^{m} m ! \widetilde{F}_{m}^{(k-m)}(\zeta)+(-1)^{m} \frac{(m+1) !}{1 !}(c-z) \widetilde{F}_{m+1}^{(k-m)}(\zeta) \\
& +\cdots+(-1)^{m} \frac{(n-1) !}{[n-1-m] !}(c-z)^{n-1-m} \widetilde{F}_{n-1}^{(k-m)}(\zeta) \\
& +\sum_{j=0}^{n-1} \sum_{r=0}^{n-1} D_{\zeta}^{k-m} \int_{c}^{\zeta} d s D_{z}^{m}\left\{(c-z)^{m} a_{j}^{(n)}[(G(s)-G(\zeta))(c-z)]\right\} \\
& \cdot\left(\int_{c}^{s}[G(\sigma)-G(s)]^{n-2} G^{\prime}(\sigma)\right. \\
& \left.\left.\cdot\left\{\widetilde{F}_{r}^{(r)}(\sigma)+\int_{c}^{\sigma} \widetilde{Q}_{r}(\tau, \sigma) \widetilde{F}_{r}^{(r)}(\tau) d \tau\right\} d \sigma\right]_{\zeta=z}\right) .
\end{aligned}
$$

Since

$$
r-[(n-2)-(k-m-1)] \leqq k-m,
$$

the last term can be written

$$
\sum_{r=0}^{n-1} \int_{c}^{z} \widetilde{F}_{r}^{(k-m)}(\sigma) \widetilde{Q}_{k-m, r}^{*}(\sigma, z) d \sigma
$$

where $\widetilde{Q}_{k-m, r}^{*}(\sigma, \zeta)$ are analytic functions of $\sigma$ and $\zeta$ for $\sigma$ and $\zeta$ in $\Omega \cup \kappa \cup \hat{\Omega}$. Introducing (4.46) into (4.45) gives an expression of the same form as (4.35). We now proceed exactly as in Lemma 4 and find that:

$$
\widetilde{F}^{(k-m)}(z)=\int_{c}^{z} \widetilde{\Gamma}_{k-m}^{*}(t, z) \widetilde{A}^{*}(t) d t+\widetilde{R}^{*}(z), \quad n \leqq m \leqq k,
$$

where

$$
\begin{aligned}
\widetilde{F}^{(k-m)}(z) & =\left(\widetilde{F}_{0}^{(k-m)}(z), \widetilde{F}_{1}^{(k-m)}(z), \cdots, \widetilde{F}_{n-1}^{(k-m)}(z)\right) \\
\widetilde{A}^{*}(z) & =\left(\int_{c}^{z}(z-t)^{n-2} \alpha_{k}^{*}(t) d t, \int_{c}^{z}(z-t)^{n-3} \alpha_{k-1}^{*}(t) d t, \cdots, \alpha_{k-(n-1)}^{*}(z)\right)
\end{aligned}
$$

and $\widetilde{\Gamma}^{*}(t, z)$ is an analytic function of $t$ and $z$ for $t$ and $z$ in $\Omega \cup \kappa \cup \hat{\Omega}$ and $\widetilde{R}^{*}(z)$ is an analytic function of $z$ for $z$ in $\Omega \cup \kappa \cup \hat{\Omega}$. But for $n \leqq m \leqq k, 0 \leqq r \leqq n-1$, we have $r-[(n-2)-(k-m-1)] \leqq$ $k-m$, thus (4.45) becomes replaced in this case by

$$
\begin{aligned}
& \left.\alpha_{k-m}^{*}(z)=D_{\zeta}^{k-m} D_{z}^{m} g_{1}^{*}(z, \zeta)\right]_{\zeta=z} \\
& =\sum_{r=0}^{n-1} \int_{c}^{\zeta} \widetilde{F}_{r}^{(k-m)}(\sigma) \widetilde{Q}_{k-m, r}^{* *}(\sigma, z) d \sigma, \quad n \leqq m \leqq k,
\end{aligned}
$$

where $\widetilde{Q}_{k-m, r}^{* *}(\sigma, z)$ are analytic functions of $\sigma$ and $z$ for $\sigma$ and $z$ in $\Omega \cup \kappa \cup \hat{\Omega}$. Combining this with (4.47) gives the result. 
The condition $\Delta_{4}(z) \neq 0$ is unnecessary in this lemma since the corresponding condition is:

$$
\Delta_{4}=(-1)^{\{n(n-1)\} / 2} 2 ! 3 ! \cdots(n-1) ! \neq 0 .
$$

Next we note that we have

Lemma 5-B. For $l+m \leqq k-1$

$$
\left.D_{\zeta}^{l} \widetilde{g}_{m}(z, \zeta)\right]_{\zeta=z}=\sum_{j=0}^{n-1} \int_{c}^{z} \widetilde{\widetilde{K}}_{k-m, j}^{*}(z, t) \alpha_{k-j}^{*}(t) d t+{\widetilde{C_{k-m}}}^{*}(z)
$$

where $\widetilde{\widetilde{K}}_{k-m, j}^{*}(z, t)$ are analytic for $z$ and $t$ in $\Omega \cup \kappa \cup \hat{\Omega}$ and $\widetilde{\widetilde{C}}_{k-m}^{*}(z)$ is analytic for $\Omega \cup \kappa \cup \hat{\Omega}$.

Proof. Same as Lemma 5 using Lemma 4-B instead of Lemma 4.

As in the case of $\left(\alpha_{k}(z), \alpha_{k-1}(z), \cdots, \alpha_{k-n+1}(z)\right)^{T}$, we get analytic extension of $\left(\alpha_{k}^{*}(z), \alpha_{k-1}^{*}(z), \cdots, \alpha_{k-n+1}^{*}(z)\right)^{T}$ into $\hat{\Omega} \cup \kappa \cup \hat{\Omega}$ which are analytic initially only in $\hat{\Omega}$, and continuous on $\hat{\Omega} \cup \kappa$. The only difference is that we use the fact that $\left.\Delta_{2}(z, \xi, \eta)\right|_{\xi=1, \eta=i} \neq 0$ on $\Omega \cup \kappa \cup \hat{\Omega}$ whereas in the extension of the $\alpha_{j}^{\prime} s$ we used the fact that $\left.\Delta_{2}(z, \xi, \eta)\right|_{\xi=1, \eta=i} \neq 0$ on $\Omega \cup \kappa \cup \hat{\Omega}$.

In an analogous way we get the analytic extension of $\widetilde{F}_{r}^{(k-n-1)}(z)$ into $\Omega \cup \kappa \cup \hat{\Omega}$, which in turn gives the analytic extension of $\widetilde{B}_{r}(z)$ into $\Omega \cup \kappa \cup \hat{\Omega}$, which finally gives the extension of $\left(e_{1}^{j}, g(z)\right)$. Since by assumption $\Delta_{1} \neq 0$ this system yields the analytic continuation of $g_{1}(\zeta), g_{2}(\zeta), \cdots, g_{n}(\zeta)$ into $\Omega \cup \kappa \cup \hat{\Omega}$.

Upon introducing the extended vector functions $h(z)$ and $g(z)$ into (4.18) we get the extension of $U^{*}(z, \hat{z})$ for $z$ in $\Omega \cup \kappa \cup \hat{\Omega}$, which was given originally only for $z$ in $\Omega \cup \kappa$. And thus, the solution of (4.13) has been extended across the boundary conditions on $\kappa$ into $\Omega \cup \kappa \cup \hat{\Omega}$. This completes the proof of the theorem.

5. Applications. (A.1) Consider the situation where we are given a solution to the differential equations

$$
u_{x x}+u_{y y}+A u_{x}+B u_{y}+C u=0
$$

where $\left(u_{1}, u_{2}, \cdots, u_{n}\right)^{T}, A, B$ and $C$ are pairwise commutative constant $n \times n$ matrices in a simply connected open region $\Omega$ of the type described in 2, part of whose boundary is the analytic arc $\kappa$, and on $\kappa$ satisfies

$$
\left.u\right|_{\kappa}=\left(\varphi_{1}(z), \varphi_{2}(z), \cdots, \varphi_{n}(z)\right)
$$

where $\varphi_{1}(z), \cdots, \varphi_{n}(z)$ are functions analytic in $\Omega \cup \kappa \cup \hat{\Omega}$. Moreover let $u \in C^{\prime}(\Omega \cup \kappa)$. Then by Theorem 1 we can uniquely extend the 
solution $u$ into $\Omega \cup \kappa \cup \hat{\Omega}$ so that it is a solution in this large region and is the only one that satisfies the given conditions provided $0 \neq$ $\left|M_{0,0}\right|$ where

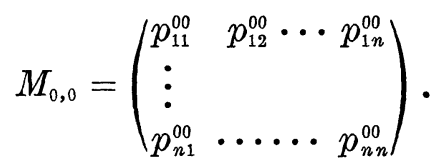

In this case

$$
p_{i j}^{00}=\begin{aligned}
& 0 \text { if } i \neq j \\
& 1 \text { if } i=j
\end{aligned} .
$$

Thus $\left|M_{0,0}\right|=1 \neq 0$ and reflection is possible.

(A.II) Theorem 2 is suitable for systems of equations of the form:

$$
\sum_{j=1}^{m} P_{i j}(\Delta) u_{j}=0 \quad i=1,2, \cdots, m
$$

where the $P_{i j}$ are polynomials with constant coefficients and $\Delta$ is the Laplacian, e.g.,

$$
\begin{array}{r}
\Delta^{2} u_{1}+a \Delta u_{1}+b u_{2}=0 \\
\Delta^{3} u_{2}+c \Delta u_{2}+d \Delta u_{1}=0
\end{array}
$$

for which if

$$
\left(w_{1}, w_{2}, w_{3}, w_{4}, w_{5}\right)^{T}=\left(u_{1}, \Delta u_{1}, u_{2}, \Delta u_{2}, \Delta^{2} u_{2}\right)^{T}
$$

then

$$
E=\left(\begin{array}{rrrrr}
0 & -1 & 0 & 0 & 0 \\
0 & a & b & 0 & 0 \\
0 & 0 & 0 & -1 & 0 \\
0 & 0 & 0 & 0 & -1 \\
0 & d & 0 & c & 0
\end{array}\right)
$$

(A.III) When the arc $\kappa$ is a portion of the $x$ axis, then condition (H.1) of Theorem 2 is automatically satisfied since then $G(z)=z$ and

$$
\begin{aligned}
\Delta_{4}(z) & =\left|\begin{array}{ccccr}
-1 & -2(c-z) & -3(c-z)^{2} & \cdots & -(n-1)(c-z)^{n-2} \\
0 & 2 ! & 3 \cdot 2(c-z) & \cdots & (n-1)(n-2)(c-z)^{n-3} \\
0 & 0 & -3 ! & \cdots & (n-1)(n-2)(n-3)(c-z)^{n-4} \\
\vdots & & & & \\
0 & 0 & 0 & \cdots & \pm(n-1) !
\end{array}\right| \\
& = \pm 1 ! 2 ! 3 ! \cdots(n-1) ! \neq 0 .
\end{aligned}
$$


(A.IV) When we consider systems of the form:

$$
\begin{aligned}
& \Delta u_{1}=a_{11} u_{1}+a_{12} u_{2} \\
& \Delta u_{2}=a_{21} u_{1}+a_{n} u_{2}
\end{aligned}
$$

$a_{i j}$ constants

then

$$
E=\left(\begin{array}{ll}
-a_{11} & -a_{12} \\
-a_{21} & -a_{22}
\end{array}\right)
$$

and condition (H.1) of Theorem 2 becomes $G^{\prime}(z) \neq 0$ for $\Omega \cup \kappa \cup \hat{\Omega}$ which is automatically satisfied because of our initial restrictions on $G(z)$.

(A.V) Given that $u_{1}$ is a solution of the metaharmonic equation

$$
\Delta^{n} u_{1}+a_{1} \Delta^{n-1} u_{1}+\cdots+a_{n-1} \Delta u_{1}+a_{n} u_{1}=0
$$

in $\Omega$ where $a_{1}, a_{2}, \cdots, a_{n}$ are constants and $u_{1}(x, y)$ is a single function, $u_{1} \in C^{2 n-2+k}(\Omega \cup \kappa) \cap C^{2 n}(\Omega), n-1 \leqq k \leqq 2 n, n \geqq 2$ and $u_{1}$ satisfies on $\kappa$ :

$$
p_{\alpha}(D) u_{1}=\sum_{r+s \leqq k} p_{\alpha 1}^{r s}(z) D_{x}^{r} D_{y}^{s} u_{1}=f_{\alpha}(z) \quad \alpha=1,2, \cdots, n
$$

where the $p_{\alpha 1}^{r s}(z)$ and $f_{\alpha}(z)$ are analytic in $\Omega \cup \kappa \cup \hat{\Omega}$. Assume that $\kappa$ is such that (H.1) is satisfied. This equation can be written as a system by letting $u_{2}=\Delta u_{1}, u_{3}=\Delta^{2} u_{1}, \cdots, u_{n}=\Delta^{n-1} u_{1}$ and equation (5.1) is equivalent to the system

Thus

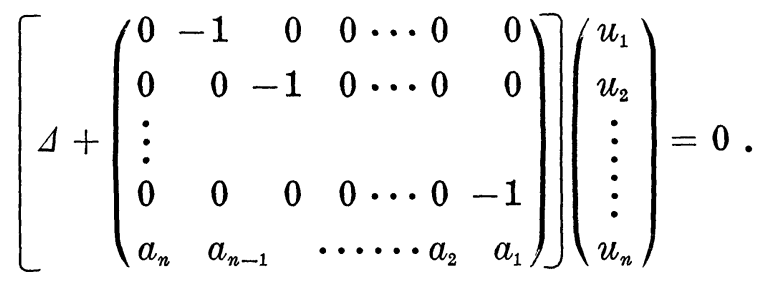

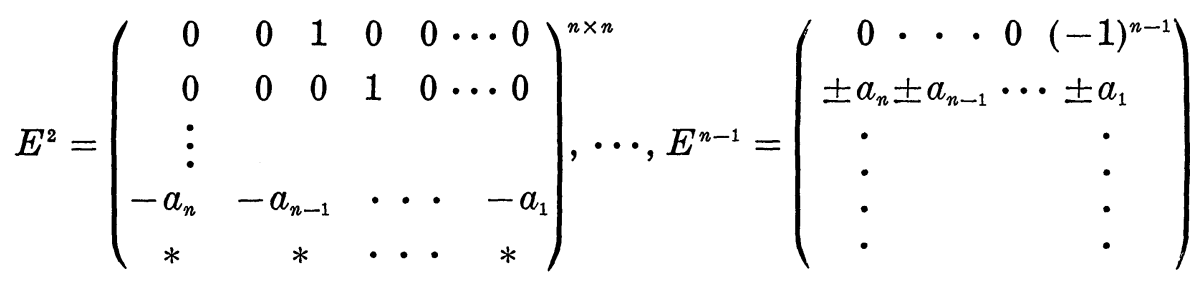

and in this case (4.14) becomes.

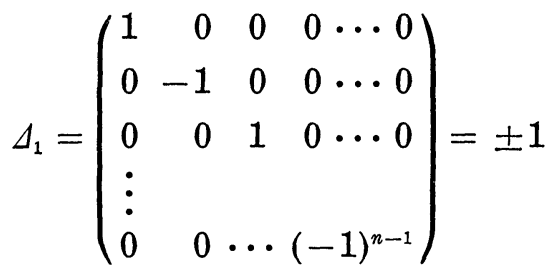


which means $\Delta_{1} \neq 0$ is not a condition in the case of the metaharmonic equation. Thus if $\Delta_{2} \neq 0$ and $\Delta_{3} \neq 0$ for $z$ in $\Omega \cup \kappa \cup \hat{\Omega}$, as given by (4.16) and (4.17), and if the $a_{j}$ are such that (H.2) is satisfied then we get that $u_{1}$ can be extended into $\Omega \cup \kappa \cup \hat{\Omega}$.

To get some idea of how we check condition (H.2) consider the example

$$
\Delta^{2} u+3 \Delta u+2 u=0 .
$$

To determine $a_{0}(s)$ and $a_{1}(s)$ of Lemma 3 where

$$
p_{i}(\lambda, s)=a_{0}(s)+a_{1}(s) \lambda,
$$

note that

$$
|E-\lambda I|=\lambda^{2}-3 \lambda+2 \lambda=(\lambda-1)(\lambda-2)
$$

and thus

$$
\begin{aligned}
& p_{1}(1, s)=a_{0}(s)+a_{1}(s)=J_{0}[\sqrt{s}] \\
& p_{1}(2, s)=a_{0}(s)+2 a_{1}(s)=J_{0}[\sqrt{2 s}] .
\end{aligned}
$$

Thus

Thus

$$
\begin{aligned}
& a_{1}(s)=J_{0}[\sqrt{2 s}]-J_{0}[\sqrt{s}] \\
& a_{0}(s)=2 J_{0}[\sqrt{s}]-J_{0}[\sqrt{2 s}] .
\end{aligned}
$$

$$
\begin{aligned}
R[z, \zeta ; t, \sigma]= & a_{0}\{(t-z)[G(\sigma)-G(\zeta)]\}\left(\begin{array}{ll}
1 & 0 \\
0 & 1
\end{array}\right) \\
& +a_{1}\{(t-z)[G(\sigma)-G(\zeta)]\}\left(\begin{array}{rr}
0 & -1 \\
2 & 3
\end{array}\right)
\end{aligned}
$$

and the representation of the solution (4.18) becomes:

$$
\begin{aligned}
U_{1}^{*}(z, \widehat{z})= & h_{1}(z)+g_{1}(\widehat{z})-a_{0}\{(c-z)[G(c)-G(\widehat{z})]\} h_{1}(c) \\
& +a_{1}\{(c-z)[G(c)-G(\widehat{z})]\} h_{2}(c) \\
- & {[G(c)-G(\widehat{z})]]_{c}^{z}\left[a_{0}^{(1)}\{(t-z)[G(c)-G(\widehat{z})]\} h_{1}(t)\right.} \\
& \left.-a_{1}^{(1)}\{(t-z)[G(c)-G(\widehat{z})]\} h_{2}(t)\right] d t \\
- & (t-z) \int_{c}^{\hat{z}}\left[a_{0}^{(1)}\{(c-z)[G(\sigma)-G(\hat{z})]\} g_{1}(\sigma)\right. \\
& \left.-a_{1}^{(1)}\{(c-z)[G(\sigma)-G(\widehat{z})]\}\right] g_{2}(\sigma) G^{\prime}(\sigma) d \sigma .
\end{aligned}
$$

In this case, the condition (H.2) becomes:

$$
\left|\begin{array}{cc}
1 & 0 \\
a_{0}^{(1)}(0) & a_{1}^{(1)}(0)
\end{array}\right|=a_{1}^{(1)}(0)=-\frac{1}{4} \neq 0,
$$


and is thus satisfied. Note that in this example (H.1) is also satisfied, since as a special case of (A.IV) it is simply $G^{\prime}(z) \neq 0$ for $z$ in $\Omega \cup \kappa \cup \hat{\Omega}$.

Note that the polyharmonic equation is a special case of the metaharmonic equation.

(A.VI) It is of interest to note that in the case of the polyharmonic equation viz. $\Delta^{n} u=0, E$ is of the form

$$
E=\left(\begin{array}{rrrrrr}
0 & -1 & 0 & 0 & \cdots & 0 \\
0 & 0 & -1 & 0 & \cdots & 0 \\
\vdots & & & & \\
0 & 0 & 0 & 0 & \cdots & -1 \\
0 & 0 & 0 & 0 & \cdots & 0
\end{array}\right) .
$$

Thus $E^{n}=0$ and the Riemann function is only the finite sum:

$$
R\left[z^{0}, \zeta^{0}, z, \zeta\right]=J_{0}\left[2 \sqrt{E\left(z-z^{0}\right)\left(G(\zeta)-G\left(\zeta^{0}\right)\right)}\right],
$$

where

$$
J_{0}[\sqrt{Q}]=I-\frac{Q}{2^{2}}+\frac{Q^{2}}{2^{4}(2 !)^{2}}-\cdots+(-1)^{n-1} \frac{Q^{n-1}}{2^{2(n-1)}[(n-1) !]^{2}} \cdot
$$

Note that the $a_{j}(s)$ of Lemma 3 are given in this case by

$$
a_{j}(s)=(-1)^{j} 2^{-2 j}(j !)^{-2} s^{j}, \quad 0 \leqq j \leqq n-1 .
$$

Thus condition (H.2) is clearly satisfied automatically. Thus for the representation (4.20) of the first component

$$
\begin{aligned}
\omega(z, \widehat{z})= & h_{1}(z)-\sum_{j=1}^{n-1} j b_{j}[G(c)-G(\widehat{z})]^{j} \int_{c}^{z}(t-z)^{j-1} h_{j+1}(t) d t \\
& +g_{1}(\hat{z})-\sum_{j=1}^{n-1} j b_{j}(c-z)^{j} \int_{c}^{\hat{z}} G^{\prime}(\sigma)[G(\sigma)-G(\widehat{z})]^{j-1} g_{j+1}(\sigma) d \sigma
\end{aligned}
$$

where

$$
b_{j}=\frac{1}{2^{2 j}(j !)^{2}}
$$

Let

$$
\begin{array}{ll}
\varphi_{j}(z)=-j b_{j} \int_{c}^{z}(t-z)^{j-1} h_{j+1}(t) d t, & j=1,2, \cdots, n-1 \\
\Psi_{j}(\hat{z})=-j b_{j} \int_{c}^{\hat{z}} G^{\prime}(\sigma)[G(\sigma)-G(\hat{z})]^{j-1} g_{j+1}(\sigma) d \sigma, & j=1,2, \cdots, n-1
\end{array}
$$

and

$$
\varphi_{0}(z)=h_{1}(z), \quad \Psi(\widehat{z})=g_{1}(\widehat{z}),
$$


then the representation (5.3) becomes:

$$
\omega_{1}(z, \widehat{z})=\sum_{j=0}^{n-1}[G(c)-G(\widehat{z})]^{j} \varphi_{j}(z)+\sum_{j=0}^{n-1}(c-z)^{j} \Psi_{j}(\widehat{z})
$$

which is an equally good representation since the $h^{\prime} s$ and $g^{\prime} s$ can be obtained simply by differentiation of the $\varphi^{\prime} s$ and $\Psi^{\prime} s$ if we utilize (H.1). This is a generalization of the representation formula of the author [8] for the biharmonic equation.

(A.VII) Next we shall check that the results of [8] for the biharmonic equation are a special case of Theorem 2 . In this case $\Delta^{2} u=0,1 \leqq k \leqq 3, \alpha_{0}^{r s}=1, \alpha_{1}^{0 k}=-k, \alpha_{1}^{1, k-1}=-(k-2), \alpha_{1}^{2, k-2}=-(k-4)$, $\alpha_{1}^{r s}=(k-2 r)=r-s$. Thus condition (4.16) and (4.17) become the same; viz.:

$$
0 \neq \Delta_{2}=\Delta_{3}=\left|\begin{array}{cc}
\sum_{r+s=k}(i)^{s} p_{11}^{r s}(z) & \sum_{r+s=k}(i)^{s}(r-s) p_{11}^{r s}(z) \\
\sum_{r+s=k}(i)^{s} p_{21}^{r s}(z) & \sum_{r+s=k}(i)^{s}(r-s) p_{21}^{r s}(z)
\end{array}\right|
$$

which is precisely the condition of [8]. As seen in (A.IV), (H.1) is satisfied and as seen in (A.VI), (H.2) is automatically satisfied and as seen in (A.V) $\Delta_{1} \neq 0$. And in this special case our theorem reduces to the theorem of [8], but with the continuity requirements strengthened by insisting that $u \in C^{k+2}(\Omega \cup \kappa) \cap C^{4}(\Omega)$ instead of only

$$
u \in C^{k}(\Omega \cup \kappa) \cup C^{4}(\Omega) \cap C^{2}(\Omega \cup \kappa)
$$

as in [8].

\section{BIBLIOGRAPHY}

1. R. D. Brown, Reflection laws of fourth order elliptic differential equations in two independent variables, J. Math. Mech. 13 (1964).

2. V. Filipenko, On the reflection of harmonic functions and of solutions of the wave equation, Pacific J. Math. 14 (1964).

3. P. Garabedian, Partial Differential Equations, Johh Wiley and Sons, Ihc., New York 1964.

4. - Analyticity and reflection of plane elliptic systems, Comm. Pure Appl. Math. 14 (1961).

5. J. Leray, Calcul, par reflexions, des fonctions $M$-harmoniques dans une bande plane verifiant aux bords $M$ conditions differentielles, a coefficients constants, Archiwum Mechaniki Stosowaj (5) 16 (1964).

6. H. Lewy, On the reflection laws of second order differential equations in two independent variables, Bull. Amer. Math Soc. 65 (1959).

7. - On the extension of harmonic functions in three variables, J. Math. Mech. 14 (1965).

8. J. M. Sloss, Reflection of biharmonic functions across analyticb oundary conditions with examples, Pacific J. Math. 13 (1963). 
Received November 9, 1966. The author wishes to gratefully acknowledge partial support for this research by the National Aeronautics Space Administration NASA Grant NGR 05-010-008. Reproduction in whole or in part is permitted for any purpose of the United States Government.

UNIVERSITY OF CALIFORNIA

SANTA Barbara, CALIFORNIA 



\section{PACIFIC JOURNAL OF MATHEMATICS}

\section{EDITORS}

\author{
H. ROYDEN \\ Stanford University \\ Stanford, California
}

\author{
J. P. JANS \\ University of Washington \\ Seattle, Washington 98105
}

J. DugundJI

Department of Mathematics

Rice University

Houston, Texas 77001

RICHARD ARENS

University of California

Los Angeles, California 90024

\section{ASSOCIATE EDITORS}
E. F. BECKENBACH
B. H. NeumanN
F. WOLF
K. YOSIDA

\section{SUPPORTING INSTITUTIONS}

\author{
UNIVERSITY OF BRITISH COLUMBIA \\ CALIFORNIA INSTITUTE OF TECHNOLOGY \\ UNIVERSITY OF CALIFORNIA \\ MONTANA STATE UNIVERSITY \\ UNIVERSITY OF NEVADA \\ NEW MEXICO STATE UNIVERSITY \\ OREGON STATE UNIVERSITY \\ UNIVERSITY OF OREGON \\ OSAKA UNIVERSITY \\ UNIVERSITY OF SOUTHERN CALIFORNIA
}

\author{
STANFORD UNIVERSITY \\ UNIVERSITY OF TOKYO \\ UNIVERSITY OF UTAH \\ WASHINGTON STATE UNIVERSITY \\ UNIVERSITY OF WASHINGTON \\ AMERICAN MATHEMATICAL SOCIETY \\ CHEVRON RESEARCH CORPORATION \\ TRW SYSTEMS \\ NAVAL ORDNANCE TEST STATION
}

\footnotetext{
Mathematical papers intended for publication in the Pacific Journal of Mathematics should be in typed form or offset-reproduced, double spaced with large margins. Underline Greek letters in red, German in green, and script in blue. The first paragraph or two must be capable of being used separately as a synopsis of the entire paper. It should not contain references to the bibliography. Manuscripts may be sent to any one of the four editors. All other communications to the editors should be addressed to the managing editor, Richard Arens, University of California, Los Angeles, California 90024.

Each author of each article receives 50 reprints free of charge; additional copies may be obtained at cost in multiples of 50 .

The Pacific Journal of Mathematics is published monthly. Effective with Volume 16 the price per volume (3 numbers) is $\$ 8.00$; single issues, $\$ 3.00$. Special price for current issues to individual faculty members of supporting institutions and to individual members of the American Mathematical Society: $\$ 4.00$ per volume; single issues $\$ 1.50$. Back numbers are available.

Subscriptions, orders for back numbers, and changes of address should be sent to Pacific Journal of Mathematics, 103 Highland Boulevard, Berkeley 8, California.

Printed at Kokusai Bunken Insatsusha (International Academic Printing Co., Ltd.), 7-17, Fujimi 2-chome, Chiyoda-ku, Tokyo, Japan.

PUBLISHED BY PACIFIC JOURNAL OF MATHEMATICS, A NON-PROFIT CORPORATION

The Supporting Institutions listed above contribute to the cost of publication of this Journal, but they are not owners of publishers and have no responsibility for its content or policies.
} 


\section{Pacific Journal of Mathematics}

\section{Vol. 24, No. $3 \quad$ July, 1968}

Duane W. Bailey, On symmetry in certain group algebras ............ 413

Lawrence Peter Belluce and Surender Kumar Jain, Prime rings with a one-sided ideal satisfying a polynomial identity ................ 421

L. Carlitz, A note on certain biorthogonal polynomials ............. 425

Charles O. Christenson and Richard Paul Osborne, Pointlike subsets of a manifold ......................................... 431

Russell James Egbert, Products and quotients of probabilistic metric

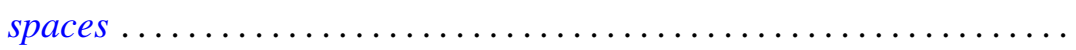

Moses Glasner, Richard Emanuel Katz and Mitsuru Nakai, Bisection into small annuli ..................................... 457

Karl Edwin Gustafson, A note on left multiplication of semigroup

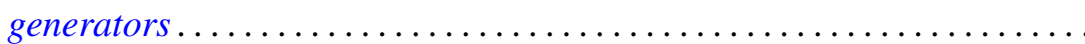

I. Martin (Irving) Isaacs and Donald Steven Passman, A characterization of groups in terms of the degrees of their characters. II ............. 467

Howard Wilson Lambert and Richard Benjamin Sher, Point-like

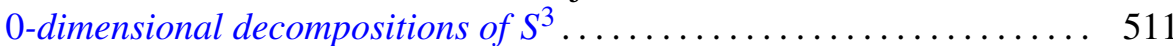

Oscar Tivis Nelson, Subdirect decompositions of lattices of width two ..... 519

Ralph Tyrrell Rockafellar, Integrals which are convex functionals . . . . . . . 525

James McLean Sloss, Reflection laws of systems of second order elliptic differential equations in two independent variables with constant coefficients ...

Bui An Ton, Nonlinear elliptic convolution equations of Wiener-Hopf type in a bounded region

Daniel Eliot Wulbert, Some complemented function spaces in $C(X)$

Zvi Ziegler, On the characterization of measures of the cone dual to a generalized convexity cone. 